\title{
Uma Arquitetura Operacional Extensível para Ferramentas de Gerenciamento de Redes
}

\author{
Rogério Borges Mouro
}

Orientador: Prof. Dr. Edson dos Santos Moreira

Dissertação a ser apresentada ao
Instituto de Ciências Matemáticas de São
Carlos, Universidade de São Paulo, como parte
dos requisitos para obtenção do título de Mestre
na área de Ciências de Computação e
Matemática Computacirnı1

São Carlos

Junho de 1997 
Universidade de São Paulo

Instituto de Ciências Matemáticas de São Carlos

São Carlos, junho de 1996 
Para Meus Pais,

Gerson e Neuza.

Sem os quais algo e alguém não existiriam.

Para Minha Esposa,

Elza.

Pelo seu amor e compreensão. 


\section{Agradecimentos}

Ao Prof. Dr. Edson dos Santos Moreira, pela orientação e dedicação.

Aos amigos Adriano Mauro Cansian e Fábio Teruo Morishita, pelo grande apoio e incentivo.

Aos amigos do ICMSC, especialmente ao Mário, Flávio, Rogério, Gustavo, Cláudia, Rejane, Onofre e André.

Aos amigos do Laboratório Intermídia, em especial o Antônio Marcos (Shiro), Robson, Roger, Manzato, Márcio (Taboca), Vidigal, Elder (Leo), Pissioli e Laura, pelas longas horas de companheirismo.

Aos companheiros de república, Tchelo, Hasegawa e Mauro pela amizade, convívio e presteza.

Aos funcionários do ICMSC, em especial à Bete, Laura, Paulinho, Eduardo, Cabral, Sônia, Ângelo e Sandra. Pela dedicação, amizade e serviços prestados.

À Fapesp, Fundação de Amparo à Pesquisa do Estado de São Paulo pela credibilidade e apoio financeiro.

Aos meus pais e à minha esposa pelo amor e incentivo em todos os dias de minha vida.

À todos que, embora não tenham seus nomes citados aqui, contribuíram de forma decisiva para a realização deste trabalho...

À Deus pelos bons momentos e pela força para superar os obstáculo. 


\section{Resumo}

O avanço tecnológico em muito tem dificultado a atualização das ferramentas de gerenciamento de redes, principalmente por não existirem propostas concretas para uma arquitetura operacional. Este trabalho tem o propósito de suprir tais deficiências, apresentando uma avançada arquitetura operacional para sistemas de gerenciamento de redes, que possui como características a facilidade e flexibilidade de incorporação de novas tecnologias e adequação a diferentes plataformas.

Os conceitos apresentados foram aplicados ao protótipo da nova ferramenta de gerenciamento de redes MultView, estendendo suas capacidades operacionais. Para tal foi dada especial atenção às tecnologias que apresentam-se como tendência, tais como Java, HTTP, CORBA, SSL, SNMP, entre outras.

O protótipo foi projetado para ser o núcleo de um sistema de gerenciamento de redes com recursos de adição, remoção e controle de módulos de aplicação em tempo real. Para tal foi definida uma interface de aplicação para ser utilizada como base na criação dos módulos a serem integrados ao sistema. A gestão das atividades de controle operacional é feita através de uma interface própria, codificada em HTML, e que pode ser acessada local ou remotamente com ajuda de um navegador Web qualquer. A ferramenta foi inteiramente codificada em linguagem Java, utilizando-se o pacote de desenvolvimento de domínio público JDK 1.0.2, nas plataformas Solaris e Windows NT. 


\section{Abstract}

Technological advances have contributed to make difficult upgrades of network management tools, mainly due to the fact that there are not concrete proposal for operational architecture in this field. This work has as goal eliminate such deficiency by presenting an advanced operational architecture for network management systems, that has as main features the easiness and flexibility for the incorporation of new technologies and the porting to different platforms.

The concepts proposed were applied to the prototype of the new MultView network management tool, extending its operational capabilities. Moreover, it was take special attention to technological trends, as Java, HTTP, CORBA, SSL, SNMP and others.

The prototype was designed to be the kernel of a network management system that has on-line capabilities to add, remove and take control of applications modules. To that, was defined an application program interface that must be used to implement applications modules to be integrated in the system environment. The control activities management are made through an HTMLcoded interface that can be handled local or remotely with any Web Browser. The tool was entirely coded in Java language, using the JDK 1.0.2 public domain package over Solaris and Windows NT platforms. 


\section{Índice}

1. INTRODUÇÃ

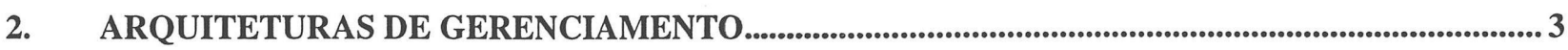

2.1 HISTÓRICO

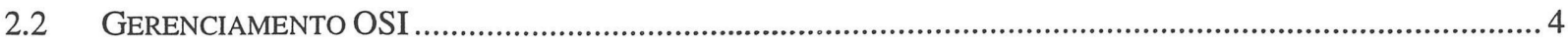

2.2.1 OSI Management Framework .................................................................................................... 4

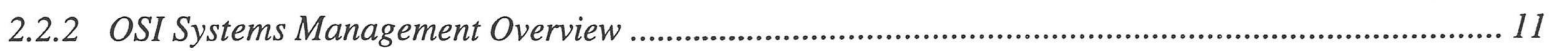

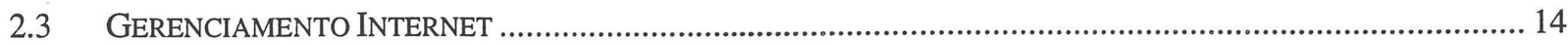

2.3.1 Modelo de Gerenciamento ............................................................................................................ 15

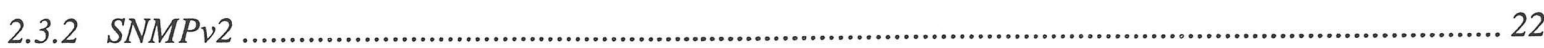

3. TECNOLOGIA DO GERENCIAMENTO.................................................................................................... 28

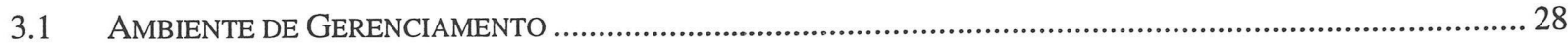

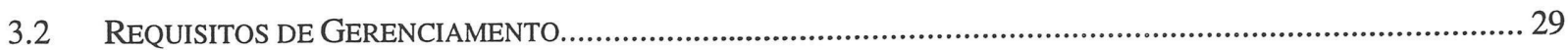

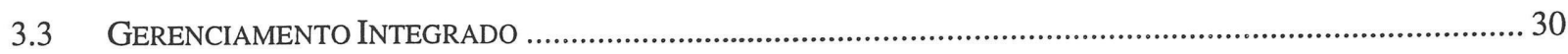

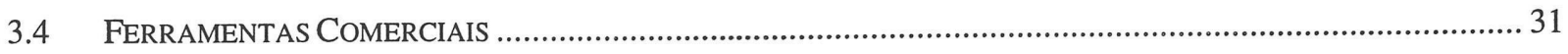

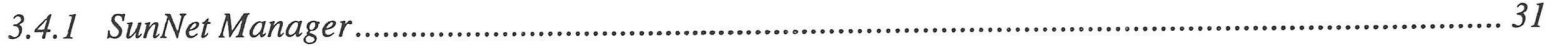

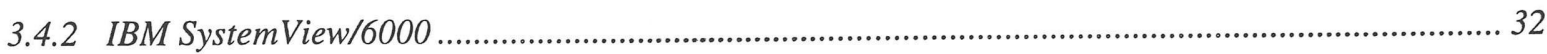

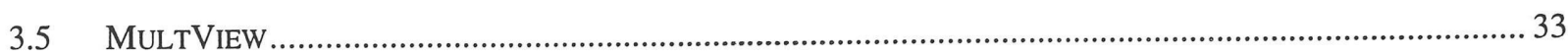

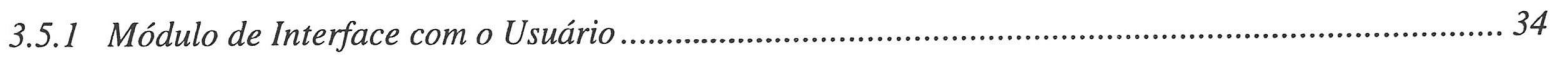

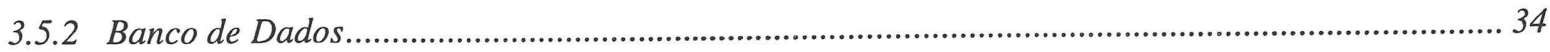

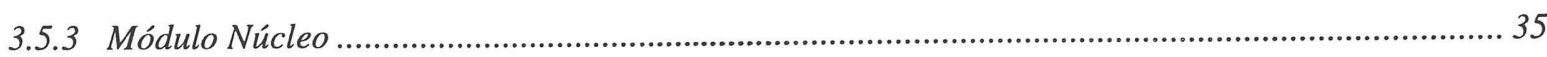

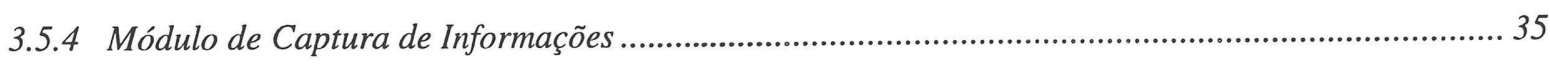

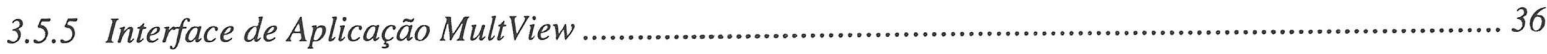

4. UMA NOVA ARQUITETURA PARA A FERRAMENTA MULTVIEW ............................................ 37

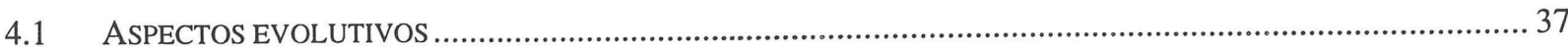

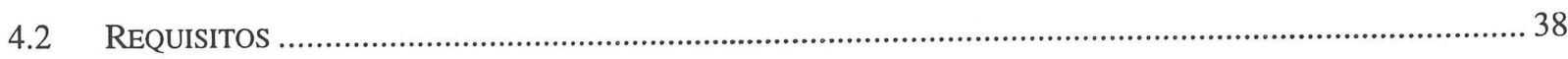

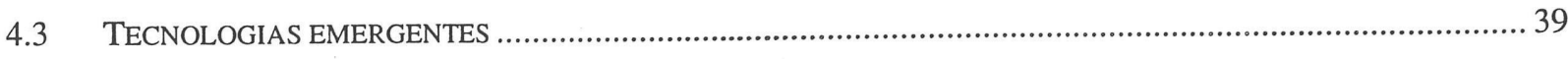

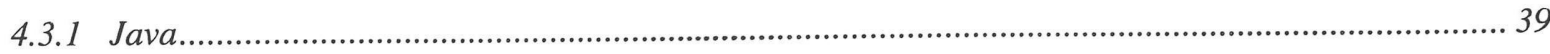

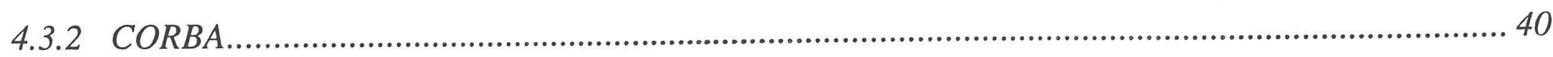

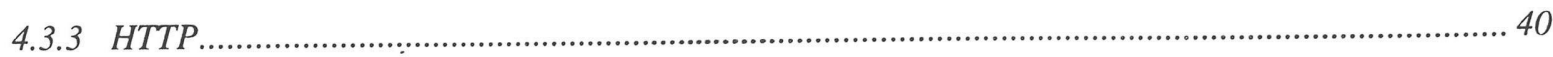

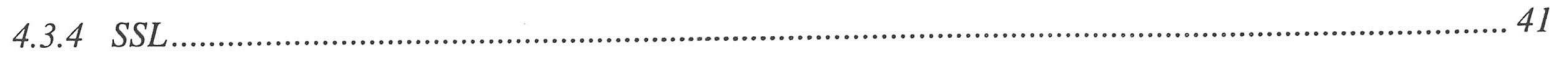

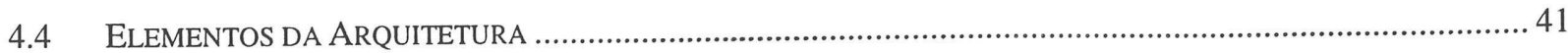

4.4.1 Camada de Módulos de Programas de Aplicação ............................................................................. 43 
4.4.2 Camada de Interface de Extensão de Aplicações 43

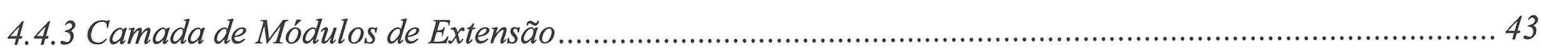

4.4.4 Biblioteca de objetos Java \& Java ORB .................................................................................4

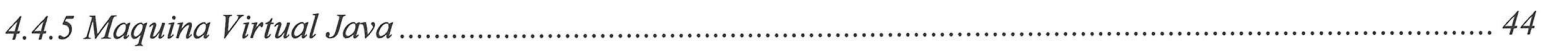

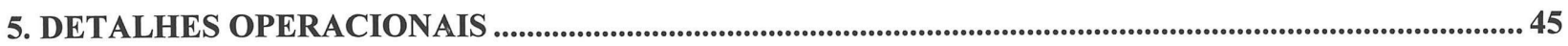

5.1 A ESTRUTURA E O RELACIONAMENTO DOS OBJETOS NO SISTEMA...........................................................45

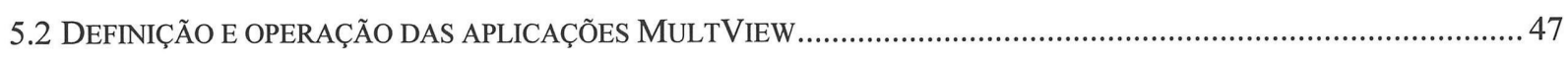

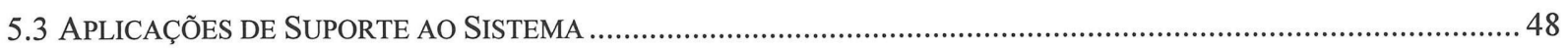

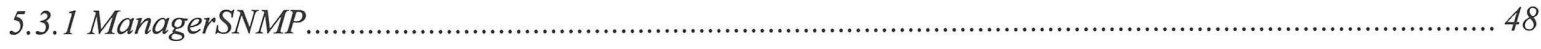

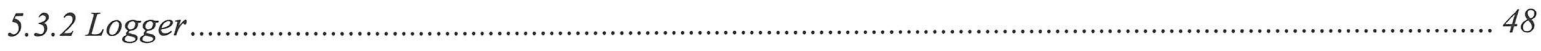

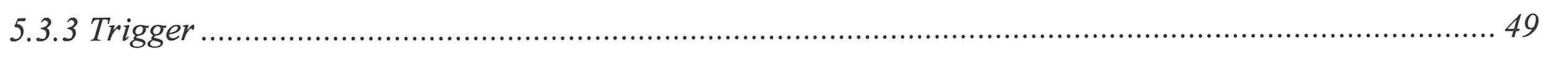

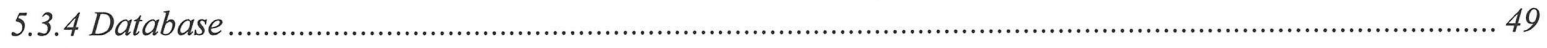

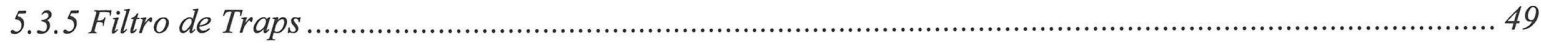

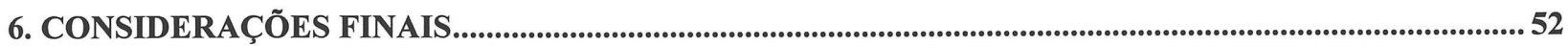

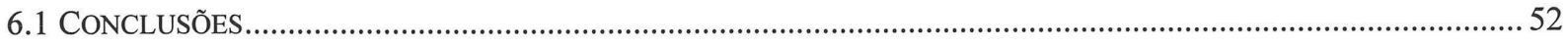

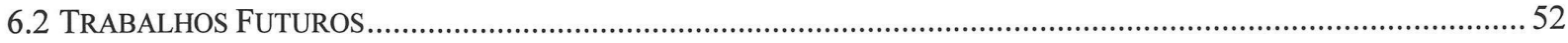

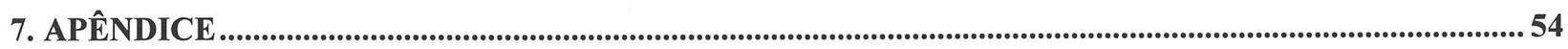

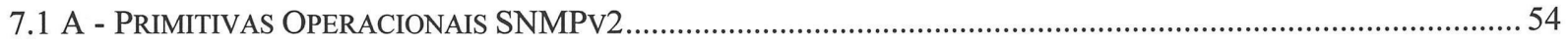

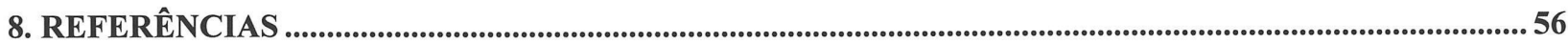




\section{Introdução}

As atividades de gerenciamento de redes de computadores tem se mostrado de grande importância à manutenção da funcionalidade e dos recursos que compõem os sistemas [Pr95]. Mais do que uma necessidade, estas atividades são indispensáveis nos cenários atuais, onde o emprego das redes de computadores extrapolou as fronteiras dos centros de pesquisa e estendeu-se aos mais diversos setores da sociedade.

Esta ampliação de seus limites operacionais culminou na necessidade de se incorporar novos conceitos e tecnologias até então excluídos dos horizontes tradicionais das redes de computadores, como a transmissão de documentos Hipermídia [Ea95], tráfego de Áudio e Vídeo codificados [Li95], e mais recentemente a tecnologia ATM [Bo95] para redes de alta velocidade, dentre outros.

Esta revolução sócio-tecnológica tem sido responsável pelo remodelamento dos padrões comportamentais [Sw96] e o surgimento de novas gerações de aplicações, trazendo consigo a necessidade de ferramentas que possibilitem o gerenciamento eficiente da complexidade introduzida pelo crescimento das redes, e a modernização dos elementos que as compõem. De fato, muitas foram as contribuições para se tentar sanar tais deficiências gerenciais. Arquiteturas de gerenciamento foram definidas pelos órgãos internacionais de padronização, como o Internet-standard Network Management Framework [Ro90, Mc91, Sc90], mais conhecido como arquitetura Internet, e o OSI Management [Is89, Is92, Is92a].

Como uma alternativa de implementação rápida, o SNMP [Sc90] (Simple Network Management Protocol) foi imediatamente absorvido pelos fabricantes de equipamentos e amplamente empregado em seus produtos, tornando-se um padrão de facto. Contudo, o SNMP logo se tornou desatualizado, apresentando várias deficiências funcionais e necessitando de uma criteriosa reavaliação. Surgiu o protocolo SNMP versão 2, mais conhecido como SNMPv2 [St93], ao qual foram incorporados recursos de segurança e a capacidade de gerenciamento descentralizado, mediante a inclusão de primitivas de comunicação gerente-gerente.

Não há dúvidas de que logo o SNMPv2 assumirá o status de padrão conquistado pela versão anterior e será utilizado por todas as plataformas. Existe, pois, a 
preocupação em fornecer ferramentas que possibilitem utilizar todos os recursos e benefícios que estão sendo incorporados ao escopo gerencial. Diversas destas ferramentas estão disponíveis no mercado e seguem de perto estas tendências, vindo de encontro às necessidades dos gerentes. Em contrapartida, muitas outras estão sendo elaboradas nos centros de pesquisa de empresas e universidades.

Como uma ferramenta de gerenciamento, o MultView [Mo94] tem se mantido em sintonia com as necessidades gerenciais e atualizado tecnologicamente, procurando antecipar tendências na área de redes. Ele é um ambiente integrado de gerenciamento baseado na arquitetura Internet, composto por vários módulos através dos quais o gerente pode interagir de forma a obter as informações necessárias às tomadas de decisões, monitorar o funcionamento dos vários elementos que compõem a rede e alterar suas configurações.

Pesquisas estão sendo desenvolvidas pelo grupo de Multimídia em Redes do Laboratório Intermídia no sentido de atualizar os recursos e, consequentemente, melhorar a funcionalidade do MultView. Este trabalho tem por objetivo promover a adequação tecnológica da ferramenta, fornecendo subsídios para facilitar extensões futuras de suas capacidades. Neste sentido, será definida e implementada uma nova arquitetura operacional, juntamente com uma aplicação exemplo: filtro de eventos.

No capítulo 2 são abordadas as arquiteturas de gerenciamento que se destacaram e contribuíram efetivamente para se estabelecer uma política de gerenciamento. Inicialmente é discutido a arquitetura de gerenciamento ISO/OSI, primeira organização a ter tomado a iniciativa e fornecido as primeiras definições. A seguir é apresentado o gerenciamento Internet que tornou-se a principal arquitetura de gerenciamento, assumindo a condição de padrão no mercado mundial; o capítulo 3 trata das necessidades gerenciais de um ambiente de redes de computadores. São apresentados as duas principais ferramentas de gerenciamento disponíveis no mercado: O SunNet Manager da Sun Microsystems e o SystemView/6000 da IBM. A ferramenta MultView recebe uma abordagem mais elaborada de sua arquitetura interna e sua funcionalidade; o capítulo 4 traz um esboço geral da proposta de adequação e atualização tecnológica a ser desenvolvida; os aspectos relevantes ao projeto e operação da ferramenta são detalhados ao longo do capítulo 5; o capítulo 6 traz as conclusões e propostas de melhoramento futuros para a ferramenta; por final, no capítulo 7 , são apresentadas as referências bibliográficas que foram utilizadas na fase de elaboração dos trabalhos e escrita da dissertação. 


\section{Arquiteturas de Gerenciamento}

\subsection{Histórico}

Ao final da década de 70 , a preocupação dos gerentes de sistemas com o crescimento e a diversidade dos elementos que compunham as redes tornaram-se bem evidenciadas. As técnicas de gerenciamento, até então baseadas em procedimentos ad-hoc, tinham como principais ferramentas as aplicações tradicionais do sistema operacional UNIX, tais como ping, traceroute, $e$ ifconfig [Co94]. Atentos a esta preocupação, grupos de pesquisa ligados a área de redes de computadores começaram a desenvolver trabalhos no sentido de estabelecer diretivas e ferramentas que viessem a suprir as deficiências no campo do gerenciamento.

Um primeiro esforço foi iniciado pela ISO como parte de seu programa Open System Interconnection (OSI) no sentido de estabelecer uma arquitetura de gerenciamento de redes. A primeira proposta para tal arquitetura surgiu no início da década de 80 e desde então sofreu um processo de evolução, culminando atualmente nos padrões OSI Management Framework, OSI System Management Overview..

De fato, a perspectiva de crescimento da Internet foi um dos fatores que mais contribuíram às iniciativas de se definir arquiteturas de gerenciamento de redes. Inicialmente o IAB (Internet Architecture Board) planejava empregar a abordagem de gerenciamento OSI. Contudo o tamanho da Internet havia atingido níveis nos quais o gerenciamento tornara-se indispensável e a arquitetura de gerenciamento OSI ainda se encontrava em processo de discussão. Esta indefinição levou o IAB a requisitar ao IETF (Internet Engineering Task Force) a definição de um protocolo de gerenciamento. O SNMP foi finalizado em meados de 1988 e teve ampla aceitação por parte dos fabricantes, apesar das deficiências que este apresentava. Em 1993 foi feito uma reavaliação do protocolo para se tentar eliminar tais deficiências e uma versão melhorada do protocolo SNMP (SNMPv2) foi definida e encontra-se em fase de implantação. 


\subsection{Gerenciamento OSI}

O primeiro padrão a fazer referência à arquitetura de gerenciamento OSI foi o $O S I$ Reference Model [Pr95], que identificava a área de gerenciamento como um importante tópico para futuras abordagens e forneceu suas primeiras definições. Por volta de 1980 foi formado um Grupo de Trabalho especial dentro da ISO, responsável pelo desenvolvimento do OSI Management. O primeiro resultado gerado por este grupo foi o OSI Management Framework que, apesar de ter consumido um longo tempo, não foi prontamente aceito como padrão adequado. Ficou então estabelecido que seria necessário produzir um documento complementar, atualmente conhecido como System Management Overview (SMO). Estes dois padrões formam a base do Gerenciamento OSI e serão abordados em maiores detalhes nas seções subseqüentes.

\subsubsection{OSI Management Framework}

O OSI Management Framework é o documento responsável por prover as definições necessárias ao estabelecimento do ambiente, da estrutura, dos serviços e dos protocolos de gerenciamento que venham a atender de forma eficiente e completa os requisitos dos ambientes gerenciais sob arquitetura OSI. Faremos a seguir uma abordagem simplista destas definições.

\subsubsection{Ambiente de Gerenciamento OSI}

O Ambiente de Gerenciamento OSI consiste de ferramentas e serviços necessários ao controle e supervisão das atividades de interconexão e dos objetos gerenciáveis associados. Para que estas perspectivas sejam postas em prática, são descritos vários modelos: modelo organizacional, modelo de informação e modelo funcional. Todos estes três modelos são tratados de forma mais elucidativa como se segue.

\section{Modelo Organizacional}

O Modelo Organizacional descreve as maneiras pelas quais os sistemas que compõem o escopo de Gerenciamento OSI podem ser distribuído administrativamente entre os domínios de gerenciamento, e dentro destes. $\mathrm{O}$ modelo organizacional visto aqui possui como premissa a adoção de uma 
série de convenções. Estas convenções fazem uso do conceito de objetos abstratos que comunicam-se entre si medianteportas abstratas [K188].

Como um dos mais importantes objetos abstratos definidos, temos o domínio de gerenciamento. Este objeto é muito utilizado para se ocultar aspectos inerentes ao ambiente gerencial, das aplicações que fazem acesso ao mesmo. Desta forma pode-se assegurar que os recursos e as atividades relacionadas ao processo de gerenciamento podem ser tão transparente aos elementos não componentes do processo quanto se deseja.

Um domínio de gerenciamento pode ser decomposto em uma coleção de um ou mais sistemas gerentes, e zero ou mais sistemas gerenciados e (sub) domínios de gerenciamento. Da mesma forma, um sistema gerenciado pode ser decomposto em um ou mais objetos gerenciados. Neste caso, um objeto gerenciado é um recurso qualquer que pode ser monitorado e controlado por um ou mais sistemas de gerenciamento. Neste contexto, um sistema gerente é uma aplicação dentro de um domínio de gerenciamento responsável por efetuar as funções de monitoria e controle sobre os objetos gerenciados e/ou subdomínios de gerenciamento.

\section{Modelo de Informação}

O Modelo de Informação delineia os aspectos necessários para se definir objetos gerenciáveis e seus inter-relacionamentos, classes, atributos, ações e nomes.

De fato, as atividades de gerenciamento refletem a necessidade de manipular uma extensa quantidade de informações, mediante a utilização de um conjunto adequado de ferramentas. Desta forma uma base de dados é necessária para armazenar os dados relativos aos estados da rede, as configurações dos sistemas, históricos de ocorrência de problemas, padrões de desempenho, parâmetros de segurança e informações de precificação.

O modelo de Base de Informação de Gerenciamento (Management Information Base), mais conhecido por MIB, foi definido e a técnica de modelagem adotada para se estruturar estas informações foi a mesma 
empregada pelas definições do padrão OSI Directory, e baseia-se na proposta ANSI [An87], sumariada a seguir.

"A MIB contém as informações referentes aos objetos gerenciados que são organizados em registros, cada qual representando uma lista de atributos e seus valores associados. Estes registros são organizadas hierarquicamente em uma Árvore de Informações de Gerenciamento (AIG), e refletem a decomposição hierárquica dos objetos que estão sendo gerenciados. A estrutura da AIG é apresentada na Figura 2.1.

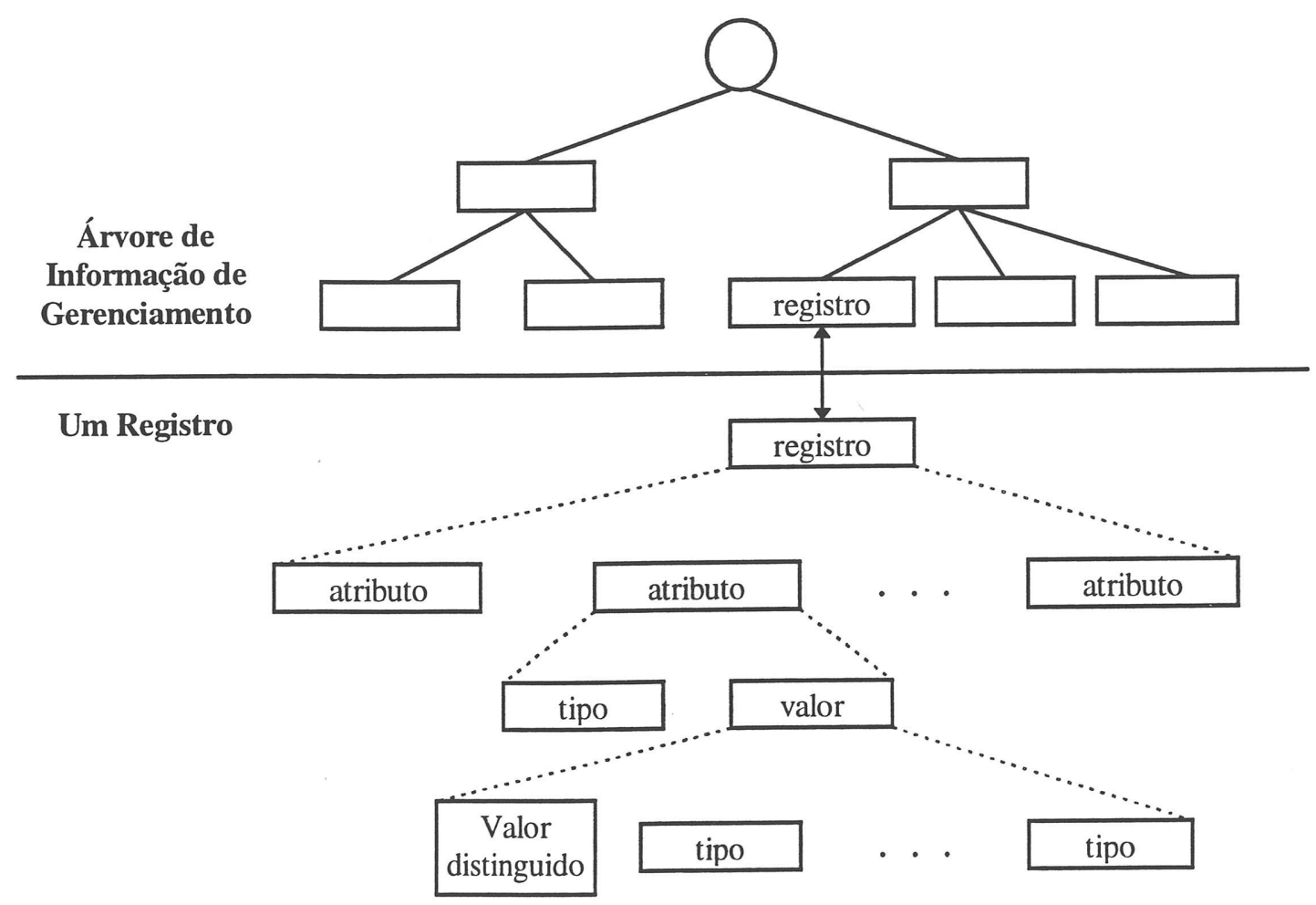

Figura 2.1 - Árvore de Informação de Gerenciamento

Objetos que apresentem características similares podem ser agrupados em Classes de Objetos, definindo-se assim cada objeto como sendo uma instância de uma determinada classe. Seguindo os conceitos do paradigma de orientação a objetos [Bo91], uma classe de objetos pode ser tratada com uma subclasse e assim herdar todas as propriedades da classe da qual é derivada (superclasse). As classes de objetos 
gerenciados são definidas mediante a atribuição das seguintes propriedades:

-Os atributos mantidos nos registros de instâncias da classe;

- As ações que podem ser invocadas para instâncias da classe;

- Os eventos que podem ser notificados por instâncias da classe;

- As subclasses das quais a classe de objetos é derivadas;

- A(s) superclasse(s) nas quais os objetos podem estar contidos."

\section{Modelo Funcional}

Um dos primeiros trabalhos de definições do OSI Management Framework já continha referências às funções que deveriam ser tratadas em uma abordagem de gerenciamento. Estas funções foram gradualmente evoluindo no que é atualmente conhecido como as cinco áreas funcionais do Gerenciamento OSI. São elas: Gerenciamento de Falhas, Gerenciamento de Configuração, Gerenciamento de Precificação, Gerenciamento de Desempenho e Gerenciamento de Segurança. Vejamos a seguir maiores detalhes de suas definições, como expostas em detalhes em [K188].

1. Gerenciamento de Falhas - É o conjunto de ferramentas responsáveis por habilitar a detecção de falhas, isolamento e correção de operações anormais. $\mathrm{O}$ gerenciamento de falha inclui funções para:

- Notificar a ocorrência de falhas;

- Registrar e manusear as notificações de eventos;

- Rastrear e identificar ocorrências de falhas;

- Agendar e executar diagnóstico e procedimentos corretivos. 
1. Gerenciamento de Configuração - É o conjunto de ferramentas responsáveis por prover o controle sobre a configuração dos elementos que compõem o sistema de rede. Este inclui funções para:

- Registrar as configurações atuais e suas eventuais alterações;

- Identificar componentes da rede.

- Ativar e desativar dispositivos de rede;

- Alterar parâmetros da rede.

2. Gerenciamento de Precificação - É um conjunto de ferramentas responsáveis por permitir o estabelecimento dos pesos e custos associados ao uso dos recursos de rede. O gerenciamento de precificação possui as funções de:

- Informar aos usuários os custos incorridos;

- Permitir o estabelecimento dos limites de contabilidade;

- Combinar custos dos vários recursos utilizados.

3. Gerenciamento de Desempenho - É um conjunto de ferramentas responsáveis por permitir o levantamento de dados estatísticos sobre a desempenho dos vários recursos da rede, para fins de otimização da Qualidade de Serviço (QoS - Quality of Service [Ku92]).

4. Gerenciamento da Segurança - É um conjunto de ferramentas que possibilitam ao gerente assegurar os recursos da rede contra as tentativas de acessos não autorizados. O gerenciamento de segurança provê suporte aos serviços de:

- Controle de acesso;

- Autenticação;

- Manutenção e manipulação dos logs. 


\subsubsection{Estrutura do Gerenciamento OSI}

O Modelo arquitetural genérico de um sistema que atua no gerenciamento OSI é observado na Figura 2.2. As atividades de gerenciamento são conseguidas mediante as funções providas pelos módulos de gerenciamento de sistemas e gerenciamento e operação de camadas. O modelo apresentado é discutido com detalhes em [K188] e recebe uma abordagem analítica em [Pr95].

\section{Gerenciamento de Sistemas}

O gerenciamento de sistemas é responsável por prover as funções necessárias ao gerenciamento dos recursos OSI e seus status através de todas as camadas da arquitetura OSI. O System Management Application Process é um processo local a um sistema, responsável por efetuar as funções de gerenciamento, que se utiliza dos serviços providos pela entidade SMAE para troca de informações de gerenciamento.

É interessante notar que o "OSI Management Framework" foca-se nas definições e aspectos relacionados à troca de informações de gerenciamento de sistemas, caracterizando assim a utilização dos protocolos da camada de aplicação para esta tarefa.

\section{Gerenciamento de Camada}

As funções do gerenciamento de camada-(N) são responsáveis por assegurar a integridade dos protocolos e permitir a alteração dos parâmetros necessários, de modo a acomodar as mudanças no ambiente e as necessidades dos usuários. Geralmente estas funções afetam as operações das entidades das camadas como um todo e não apenas determinadas instâncias.

$\mathrm{O}$ gerenciamento de camada-(N) suporta o monitoramento, controle e coordenação dos objetos gerenciáveis da camada-(N). Além disto, os protocolos de gerenciamento da camada-(N) são suportados pelos protocolos das camadas $(\mathrm{N}-1)$ e inferiores. 


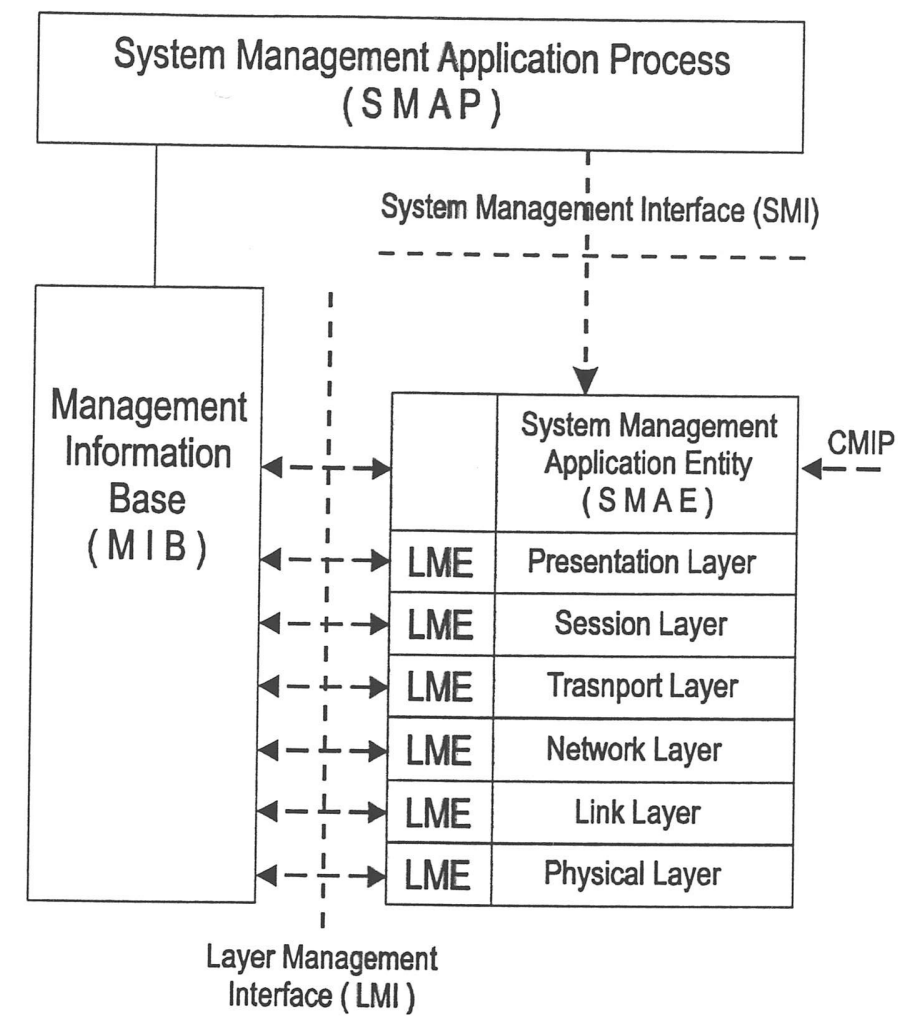

Figura 2.2 - Modelo Arquitetural de Gerenciamento OSI [K188]

\section{Operação de Camada}

A operação de camada é responsável por prover as funções de gerenciamento das instâncias de comunicação. Neste caso a informação de gerenciamento é transferida como parte do protocolo usual da camada.

\subsubsection{Serviços do Gerenciamento OSI}

Os requisitos de comunicação para as atividades de gerenciamento podem ser separados em três categorias:

1. Serviço de Manipulação de Dados - Estes serviços são responsável por prover as primitivas necessárias à manipulação das informações contidas nas bases de informação de gerenciamento (MIBs). O "OSI Common Management Information Service" (CMIS - [Is90]) define estes cinco 
serviços para tal finalidade: $M$-Confirmed-Get, M-Set, M-Confirmed-Set, M-Confirmed-Create e M-Confirmed-Delete.

2. Serviço de Sinalização de Eventos - Estes serviços são responsáveis por prover as primitivas necessárias ao anúncio de eventos importantes que possam vir a ocorrer nos elementos gerenciados. CMIS define duas primitivas que visam atender estas necessidades. São elas: $M$-Event-Report e M-Confirmed-Event-Report.

3. Serviço de Controle Direto - De modo a permitir o controle de forma direta por parte do gerente, o CMIS fornece duas primitivas que atendem esta finalidade. Estas primitivas não alteram as informações contidas nas MIBs, por outro lado eles são utilizados para requisitar que os sistemas gerenciados efetuem determinadas ações. Estas ações podem ainda alterar permanentemente o estado dos sistemas. Tais primitivas são: M-Action $e$ M-Confirmed-Action.

\subsubsection{OSI Systems Management Overview}

Tendo inicio em meados de 1987, o texto final do OSI Systems Management Overview foi finalizado em junho de 1991. Este documento, se comparado ao OSI Management Framework, possui uma quantidade maior de informações e apresentou-se com uma melhor receptividade dentro da própria instituição ISO.

Em se tratando de gerenciamento de sistemas, o SMO faz distinção entre os aspectos de Informação, organizacional, funcional e de comunicação. São descritos a seguir cada um desses aspectos.

\subsubsection{ASPECTO DE INFORMAÇÃO}

Os aspectos de informação do modelo de gerenciamento de sistemas trata dos recursos que estão sendo gerenciados. Estes recursos são vistos como "objetos gerenciados". 
Apesar de ter sido introduzido como parte do OSI Management Framework, os conceitos de objetos gerenciados vinham sofrendo diferentes interpretações. Isto acarretava em erros graves de definições e, consequentemente, de implementações. Desta forma a definição de objeto gerenciado foi refinada como se segue:

"Um objeto gerenciado é a visão do gerenciamento OSI de um recurso que esteja sujeito a gerenciamento, tal como uma entidade de camada, uma conexão ou um item de um equipamento físico de comunicação. Sendo assim, um objeto gerenciado é a abstração de um recurso que representa suas propriedades como visto pelo gerenciamento. Uma parte essencial da definição de um objeto gerenciado é o relacionamento entre estas propriedades e o comportamento operacional do recurso. De forma geral, este relacionamento não é modelado."

De acordo com o Modelo de Informação de gerenciamento OSI [Is93] a visão de gerenciamento é descrita em termos da Figura 2.3, onde os Atributos são as características ou propriedades dos objetos; as Operações são as atividades que podem ser efetuadas pelos objetos; o Comportamento reflete as respostas às operações efetuadas; e Notificações são avisos gerados em decorrência de alguma alteração dos atributos dos objetos.

\section{OBJETO GERENCIADO}

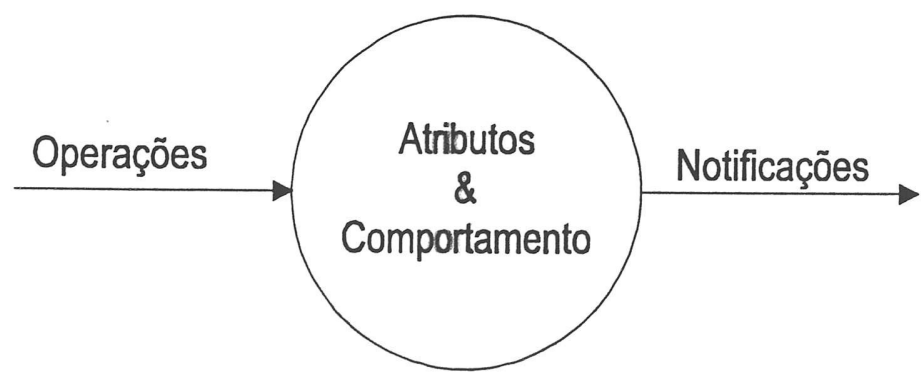

Figura 2.3 - Objeto Gerenciado

O conceito de objeto gerenciado é refinado em uma série de padrões adicionais (ver [Pr95]), que são conhecidos por Structure of Management Information standards (padrões de Estrutura de Informação de Gerenciamento), ou SMI. O padrão SMI não especifica os objetos de gerenciamento; estes são definidos pelos grupos de trabalhos responsáveis por cada camada do modelo de referência OSI. 
O gerenciamento de sistemas OSI pressupõe uma organização centralizada. Desta forma um único gerente pode exercer controle sobre vários agentes, como ilustrado na Figura. 2.4.

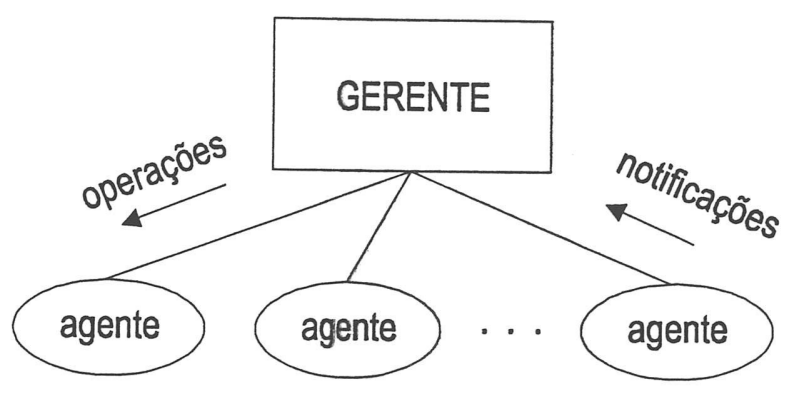

Figura 2.4 - Interação Gerente-agente

\subsubsection{AsPECTO FUNCIONAL}

De fato, o OSI Management Framework definiu cinco áreas funcionais de gerenciamento, apresentadas anteriormente. Contudo, foi observado que a maioria dos protocolos destas áreas usavam um mesmo conjunto de funções elementares de gerenciamento. Sendo assim, em 1988 ficou decidido que seriam cessadas as atividades relacionadas aos protocolos das cinco áreas funcionais e seriam concentrados esforços na definição destas funções elementares de gerenciamento. Atualmente, estas funções são conhecidas por Systems Management Functions (SMF).

\subsubsection{Aspecto de Comunicação}

OSI definiu o Common Management Information Service (CMIS - [Is90]) como o principal serviço para troca de informações de gerenciamento. O papel do CMIS é restrito à troca de informações; o controle do sistema é deixado para o MIS-user que está localizado no topo do CMIS. O padrão CMIS define as primitivas de serviço: $M$-Get, $M$-Cancel-Get, $M$-Set, $M$-Action, $M$-Create, M-Delete e M-EventReport. 


\subsection{Gerenciamento Internet}

Com a necessidade de uma abordagem estruturada e padronizada de gerenciamento Internet bem evidenciada, começaram a surgir em meados de 1987 propostas que atendiam os requisitos necessários. Uma destas, o "High-level Entity Management System/Protocol" (HEMS/HEMP [Tr87, Tr87a]) foi rapidamente descartada, permanecendo apenas duas: o SNMP e o Common Management Over TCP/IP (CMOT [Be90]). Em março de 1988 o IAB decidiu que o SNMP seria adotado como uma alternativa emergencial em curto prazo, enquanto se esperava por um amadurecimento do CMOT como uma alternativa definitiva em longo prazo.

O CMOT tinha como meta a utilização do padrão de gerenciamento OSI sobre o ambiente Internet. Com os mesmos sintomas do próprio padrão OSI de gerenciamento, o atraso na elaboração das definições e a falta de implementações, todos os trabalhos relacionados ao CMOT foram suspensos em 1992, consagrando assim o SNMP como o padrão definitivo da arquitetura de gerenciamento Internet.

O SNMP teve como embrião o Simple Gateway Management Protocol (SGMP [Da87]), que foi concebido para permitir o gerenciamento dos sistemas intermediários que tomam parte na Arquitetura Internet. De fato, o SGMP teve seu escopo de abrangência estendido de modo a comportar o gerenciamento dos demais sistemas da arquitetura.

Os princípios que regem o gerenciamento Internet podem ser delineados através das seguintes idéias:

- Todos os sistemas conectados à rede devem ser gerenciados com SNMP

- O custo de adicionar capacidade de gerenciamento aos sistemas existentes devem ser mínimos.

- Deve ser relativamente fácil a extensão das capacidades de gerenciamento dos sistemas existentes.

- O gerenciamento deve ser robusto o suficiente, de forma a possibilitar o gerenciamento dos sistemas mesmo no caso de ocorrência de falhas 
O SNMP foi aceito prontamente como um padrão de mercado para o gerenciamento de redes de comunicação de dados. Porém o SNMP foi concebido com uma série de deficiências que impõem restrições gerenciais aos ambientes atuais, como aspectos de segurança, comunicação gerente-gerente entre outros. Em 1992 foi iniciado um trabalho para se desenvolver uma versão melhorada do SNMP: o SNMPv2. Estes protocolos serão discutidos nas seções subseqüentes.

\subsubsection{Modelo de Gerenciamento}

O modelo de gerenciamento de redes de computadores baseado na classe de protocolos Internet é composto por quatro elementos principais:

- Estação de Gerenciamento (Gerente)

- Agente de Gerenciamento (Agente)

- Base de Informações de Gerenciamento (MIB)

- Protocolos de Gerenciamento de Redes

A estação de gerenciamento é o elemento responsável por prover a interação do administrador com o sistema de gerenciamento de redes. É composta por aplicações para análise e estatística dos dados, interface para monitorar e controlar os recursos da rede e uma base de dados contendo as informações extraídas das entidades gerenciadas.

Os agentes de gerenciamento são responsáveis por atenderem as requisições e executarem as tarefas designadas pela estação de gerenciamento e, além disso, podem alertar a qualquer momento a estação de gerenciamento sobre a ocorrência de eventos importantes. Estes agentes são normalmente implementados nos elementos chaves das redes, tais como bridges, routers, hubs e estações de trabalho.

A base de informações de gerenciamento é formada pelos objetos representativos dos recursos disponíveis. Estes objetos são padronizados para elementos pertencentes a uma mesma classe (i. é, bridges suportam os mesmos objetos) e são representados como variáveis. As funções de monitorização são efetuadas mediante 
a leitura do conteúdo destas variáveis e as funções de controle, através da modificação de seus conteúdos. Um tratamento mais completo sobre MIBs é feito por Stallings [St93]

O protocolo de gerenciamento de redes é responsável por efetuar a troca de informações entre os agentes e a estação gerente, e permite que sejam efetuadas as seguintes classes de operações:

- "GET" - Permite a obtenção, por parte da estação de gerenciamento, dos valores associados aos objetos da MIB;

- "SET" - Possibilita que a estação de gerenciamento efetue alterações dos valores dos objetos em um dado agente;

- "Trap" - Permite que um agente possa notificar a estação de gerenciamento sobre a ocorrência de algum vento importante.

\subsubsection{Protocolo de Gerenciamento}

O SNMP é um protocolo ao nível de aplicação projetado para operar sobre UDP [Po80], que também faz parte da classe de protocolos Internet.

Em uma estação de gerenciamento dedicada, um processo gerente controla o acesso a uma MIB central, na própria estação, e funciona como uma interface para o administrador da rede. O processo gerente executa suas funções de gerenciamento através da utilização do SNMP.

Por outro lado, em cada estação há um processo agente responsável por interpretar as mensagens SNMP e efetuar o controle necessário sobre a MIB. Este esquema de configuração é representado na Figura 2.5. Sob contexto do SNMP, a figura 2.6 que se segue permite-nos uma visão mais clara deste mecanismo de gerenciamento.

\subsubsection{EstratéGia de Sinalização(Trapping)}

Em um ambiente complexo, com uma grande quantidade de agentes, ficaria inviável, por parte da estação de gerenciamento, efetuar regularmente uma amostragem do conteúdo dos objetos contidos em cada agente. Por este motivo preferiu-se a abordagem de se identificar o estado inicial de cada elemento, e a 
seguir aguardar a notificação de uma possível alteração deste estado, enviada pelo mesmo. Esta abordagem pode ser expressa pela seguinte estratégia:

- No momento da iniciação e a intervalos freqüentes de tempo (uma vez ao dia por exemplo) a estação de gerenciamento pode efetuar a amostragem das informações principais contidas em cada um dos agentes gerenciados;

- Uma vez estabelecidas as condições iniciais de operação dos agentes, estes ficam responsáveis por sinalizar à estação de gerenciamento da ocorrência de algum evento importante (p. ex., falha de um dispositivo, condições de sobrecarga, etc.) através de uma mensagem SNMP conhecida como Trap.

- De posse da informação sobre a ocorrência de uma situação anômala, a estação pode tomar as providências necessárias

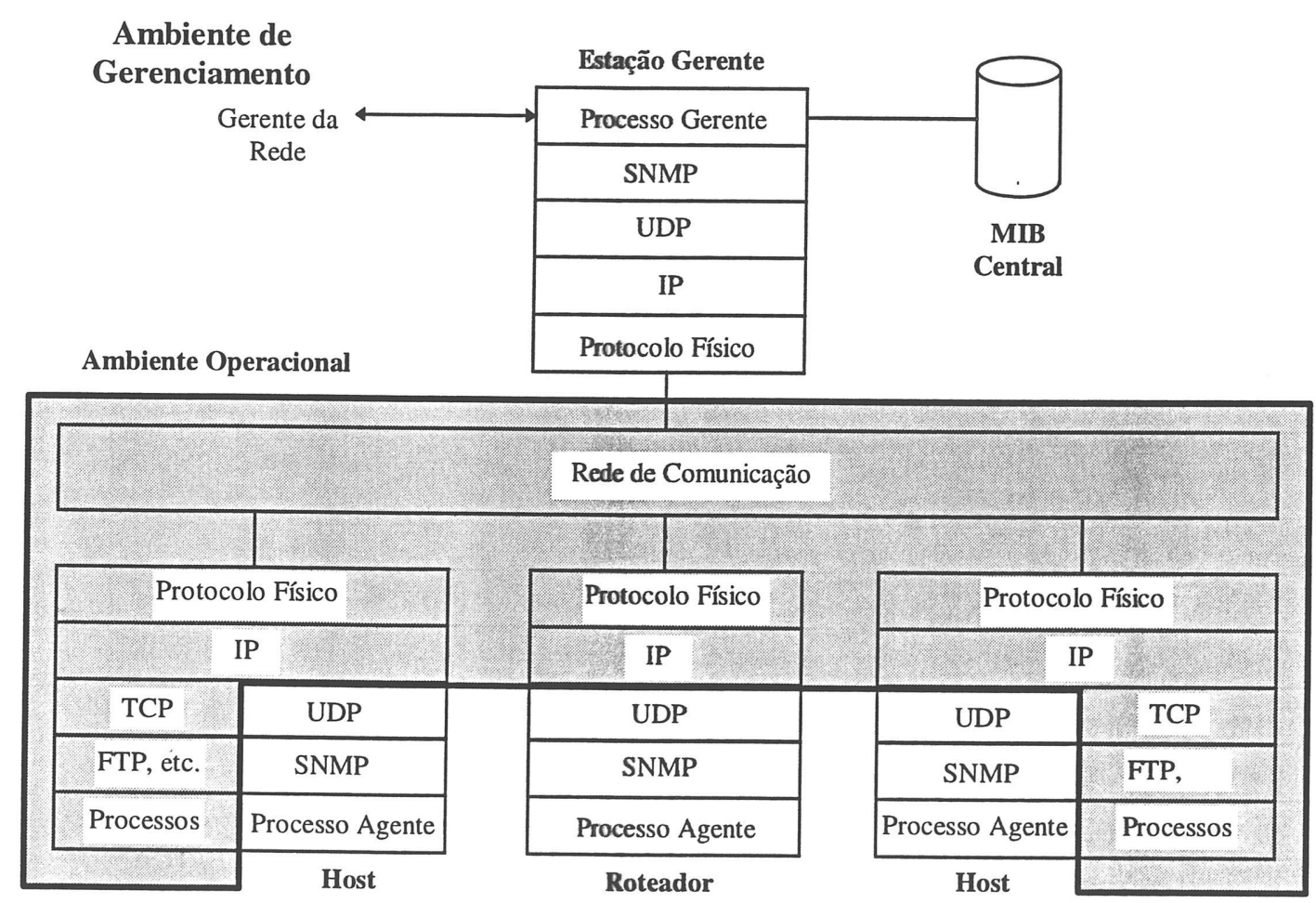

Figura 2.5 - Configuração do SNMP [St93] 
Existe toda uma discussão sobre como possibilitar o gerenciamento, através do SNMP, de elementos que implementam (ou não) outros protocolos de gerenciamento. A funcionalidade dos agentes proxies é justamente permitir o gerenciamento destes recursos por uma estação gerente SNMP, mediante a conversão e/ou ajustes entre os protocolos. Uma abordagem mais criteriosa destes elementos pode ser encontrada em [Ro91].

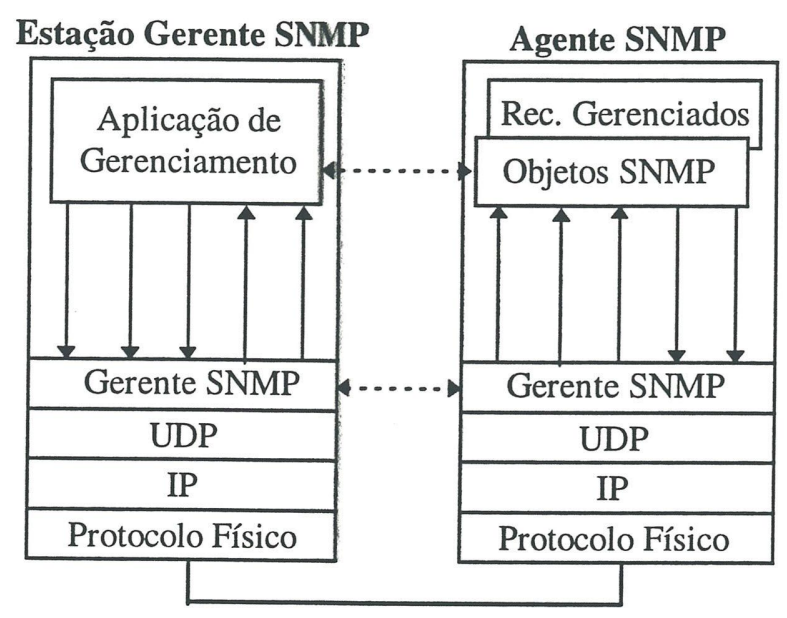

Figura 2.6 - Mecanismo de Gerenciamento SNMP [Jo90]

\subsubsection{Primitivas Operacionals}

Aqui são abordadas as características e limitações do protocolo SNMP sem, contudo, entrar em detalhes específicos do protocolo, que são cobertos em sua plenitude em [Sc90].

O SNMP possui as características típicas encontradas em aplicações distribuídas [Mu89], onde grupos não necessariamente disjuntos de agentes são gerenciados por uma estação gerente. Por outro lado, podemos identificar uma relação onde cada agente além de controlar sua MIB local ainda deve permitir o controle desta MIB por um número de estações gerentes. Esta relação de controle pressupõe a existência das três características apresentadas a seguir:

- Serviço de Autenticação - Capacidade de identificação e validação das estações gerentes autorizadas; 
- Política de Acesso - Possibilidade de estabelecer diferentes privilégios de acesso às estações de gerenciamento;

- Serviço de Proxy - Capacidade de um agente funcionar como um agente proxy intermediário para outra estação de gerenciamento.

Todos estes aspectos envolvem fortes conceitos de segurança, de modo a permitir que os agentes protejam a si e a suas MIBs contra tentativas de acessos não autorizados. O SNMP provê apenas uma primitiva e limitada capacidade de segurança. $\mathrm{O}$ conceito de comunidade.

Uma comunidade SNMP nada mais é do que uma relação de autenticação, controle de acesso e característica de proxy entre um agente e um conjunto de estações gerentes, definidos localmente nos agentes e mantidas pelas estações de gerenciamento. Uma comunidade é identificada por um nome único, sob contexto local, conhecido como nome de comunidade.

Para o serviço de autenticação, o nome de comunidade funciona como um mecanismo rudimentar, onde cada mensagem trocada entre um agente e o gerente é autenticada pela inclusão do nome de comunidade correto.

A possibilidade da definição de várias comunidades por parte do agente permite estabelecer um método de controle de acesso, onde para cada comunidade se especifica uma categoria diferente de acesso à MIB. Existem dois aspectos a serem considerados sobre este controle de acesso:

- Visão da MIB SNMP - Um subconjunto dos objetos em uma MIB;

- Modo de Acesso SNMP - "READ-ONLY" (apenas leitura) e "READWRITE” (leitura e escrita).

A combinação de uma visão da MIB e um modo de acesso é conhecida como um "Perfil de Comunidade SNMP" e permite delinear o grau de acessibilidade de uma determinada comunidade SNMP e seus respectivos gerentes. Além disso, o conceito de comunidade também é útil sob o ponto de vista do serviço proxy, onde os elementos envolvidos podem manter uma política de acesso entre as partes.

Existe todo um esquema definido para que o acesso às informações contidas nas MIBs possa ser efetuado de forma direta, eliminando-se os problemas relacionados a ambigüidades da informação. O SNMP define duas técnicas de identificação de 
instâncias de objetos: técnica de acesso seqüencial e técnica de acesso aleatório. Uma discussão mais abrangente sobre a estrutura das informações de gerenciamento e das técnicas referidas são encontradas em [Ro90] e [St93.], respectivamente.

Cada mensagem trocada entre uma estação de gerenciamento e um agente inclui o número da versão SNMP, um nome de comunidade e um dos cinco tipos de Unidades de Dados do Protocolo (PDUs), que são conhecidas como primitivas operacionais e que são apresentadas a seguir.

Para se permitir o intercâmbio de múltiplos objetos, todas as PDUs possuem um campo de conexão variável consistindo de uma seqüência de referências para instâncias de objetos, juntamente com os valores associados [Sc90].

Além disso, cada mensagem SNMP pode agrupar operações de um mesmo tipo (Get, Set, Trap), permitindo que uma estação de gerenciamento obtenha os valores de todos os objetos escalares de um mesmo grupo em um agente particular.

As primitivas operacionais SNMP são apresentadas a baixo.

- "GetRequest PDU" - Esta primitiva é direcionada pela entidade SNMP de uma estação gerente, a uma entidade SNMP de um agente, e tem como finalidade obter os valores associados aos objetos de interesse.

- "GetNextRequest PDU" - É uma primitiva muito semelhante à GetRequest PDU, e possui como única diferença o fato de que o GetResponse PDU, para cada variável requisitada, contém o valor da instância do objeto que seja o próximo na seqüência "lexicográfica". Como a GetRequest, a GetNextRequest é uma operação atômica: ou todos ou nenhum dos valores são retornados.

- "GetResponse PDU" - Caso seja possível efetuar por completo a operação requisitada, a entidade SNMP destino responde com um GetResponse PDU contendo as informações. Caso contrário esta resposta conterá um status de erro.

- "SetRequest PDU" - É uma primitiva idêntica à GetRequest, porém com a função de escrever um valor específico para uma instância de um objeto. 
- "Trap PDU" - Esta primitiva é gerada pela entidade SNMP de um agente e enviada à entidade SNMP de uma estação de gerenciamento, com o propósito de notificá-la sobre a ocorrência de um evento importante.

Ben-Artzi, Chandna e Warrier, em 1990, identificaram as potenciais limitações que recaem sobre o SNMP [Pr95]:

- SNMP pode não ser adequado ao gerenciamento de grandes redes de computadores, dado ao fato de apresentar limitações de desempenho para obtenção de informações através de requisições explícitas (polling).

- O protocolo não é adequado para a aquisição de grandes quantidades de informação.

- O SNMP provê apenas um mecanismo rudimentar de autenticação, sendo assim mais adequado ao monitoramento do que ao controle.

- Não há suporte a comandos imperativos.

- SNMP não dá suporte a comunicação gerente-gerente, sendo impossível o intercâmbio de informações entre estações gerentes.

\subsubsection{SNMP vs SNMPv2}

De fato, o SNMP se apresenta como um protocolo restrito, ineficiente e principalmente inseguro se exposto a ambientes relativamente grandes e complexos. Estas deficiências, aliadas às necessidades de gerenciamento dos recursos de redes atuais, culminaram com a evolução do protocolo para o que hoje conhecemos como SNMP versão 2 (SNMPv2).

Serão discutidas as principais características de comunicação, enfatizando-se principalmente os aspectos de comunicação gerente-gerente e mecanismos de segurança que foram incorporados ao SNMPv2. 


\subsubsection{SNMPv2}

O protocolo SNMPv2 suporta plenamente tanto uma estratégia de gerenciamento centralizada quanto distribuída, onde algumas entidades operam desempenhando um papel de gerente e agente simultaneamente.

Os avanços incorporados ao SNMPv2 podem ser classificados em quatro categorias principais expostas a seguir:

- Estrutura das informações de gerenciamento (SMI);

- Operações do Protocolo;

- Comunicação Gerente-Gerente;

- Segurança.

\subsubsection{ESTRUTURA DA INFORMAÇÃO DE GERENCIAMENTO}

A estrutura da Informação de Gerenciamento (SMI) para SNMPv2 é baseada no SMI para SNMP e possui uma especificação mais elaborada, eliminando-se ambigüidades nas definições dos objetos encontradas nas especificações anteriores em ASN.1 [Ro90 e Ro91a]. Estas alterações exercem influência direta sobre a implementação das MIBs SNMPv2 e seus esquemas de funcionamento. Os detalhes destas alterações e suas conseqüências são cobertos em detalhes por [St93] e criteriosamente documentadas nas referências citadas anteriormente.

\subsubsection{OPERAÇóES DO PROTOCOLO}

O protocolo SNMPv2 provê três tipos de operações de acessos às informações de gerenciamento:

1.Requisição/Resposta Gerente-Agente - uma entidade cumprindo papel de gerente envia uma mensagem de requisição a uma entidade agente que por sua vez atende à requisição e envia resposta. 
2.Requisição/Resposta Gerente-Gerente - uma entidade gerente envia uma requisição a uma outra entidade gerente que por sua vez responde à requisição.

3.Agente/Gerente não confirmada - Uma entidade SNMPv2 no papel de Agente envia um Trap a uma outra entidade que esteja cumprindo o papel de Gerente. Neste caso não há mensagem de resposta.

Observa-se que apenas o item 2 se apresenta como uma característica inovadora no protocolo SNMPv2, em relação ao protocolo SNMP. Além disso, existem novas primitivas operacionais que foram incorporadas ao SNMPv2. Estas primitivas são expostas a seguir. Maiores detalhes de sua operação e de como são processadas podem ser encontrados no apêndice A:

- SNMPv2 "GetRequest PDU" - esta primitiva é funcionalmente idêntica ao SNMP GetRequest PDU, diferindo-se única e exclusivamente pelo modo como a resposta é tratada.

- SNMPv2 "GetNextRequest PDU" - é idêntica em formato e semântica à primitiva "GetNextRequest PDU" do protocolo SNMP, diferenciando-se pelo fato de não ser uma operação atômica, permitindo-se o processamento de tantas variáveis quanto possíveis.

- "GetResponse PDU" - caso seja possível efetuar por completo ou parte da operação requisitada, a entidade SNMP destino responde com um GetResponse PDU contendo as informações.

- SNMPv2 "GetBulkRequest PDU" - esta primitiva representa uma das maiores melhorias no protocolo SNMPv2. Através dela é possível especificar múltiplos sucessores lexicográficos para serem selecionados, aumentando-se a eficiência do protocolo perante as requisições por grandes quantidades de informação.

- SNMPv2 "SetRequest PDU” - É idêntica em formato e semântica ao SetRequest PDU para SNMP, diferenciando-se apenas pelo modo como as respostas são tratadas.

- SNMPv2 "Trap PDU" - Semelhante em funcionalidade ao SNMP Trap $P D U$, porém diferenciando-se no formato, que se assemelha ao formato dos PDUs SNMPv2 anteriores, com exceção do GetBulkRequest PDU. 
SNMPv2 "InformRequest PDU" - É enviado por uma entidade SNMPv2 que esteja desempenhando o papel de Gerente a uma outra entidade atuando como Gerente, de modo a requisitar que a última provenha informações de gerenciamento.

\subsubsection{ComunicaÇÃo Gerente-Gerente}

Basicamente as capacidades de comunicação Gerente-Gerente adicionadas ao protocolo SNMPv2 recaem sobre a definição de objetos que compõem uma MIB específica. A MIB Gerente-Gerente, cujos objetos são responsáveis por descreverem o comportamento de uma entidade SNMPv2 agindo no papel de Gerente. Esta MIB é constituída por dois grupos:

1. Grupo de Alerta - Uma coleção de objetos que permitem a descrição e configuração dos limiares de alerta de uma entidade SNMPv2 que esteja atuando tanto como Agente quanto como Gerente;

2. Grupo de Eventos - Uma coleção de objetos que permitem a descrição e configuração dos eventos de uma entidade SNMPv2 que esteja atuando como Agente e Gerente.

O Grupo "snmpAlarm” da MIB Gerente-Gerente é responsável por estabelcer as condições de alertas. Consiste de uma simples tabela, "snmpAlarmTable", e uma variável escalar simples, "snmpAlarmNextIndex" cuja finalidade é indicar o número do próximo índice disponível para criação de "tuplas".

O grupo "snmpEventTable" é responsável por suportar as definições dos eventos e a configuração das notificações. O grupo de eventos snmpEvent consiste de duas tabelas, "snmpEventTable" e "snmpEventNotifyTable", mais objetos escalares associados.

Cada "tupla" na tabela snmpEventTable define um evento que podem ser disparados por uma condição associada na tabela snmpAlarmTable, ou através das condições definidas na macro "NOTIFICATION-TYPE" [Ca93].

A tabela snmpEventNotifyTable define as notificações que deverão ocorrer quando um evento associado for disparado. As "tuplas" desta tabela descrevem os tipos e o destino para um InformRequest PDU enviado em um evento particular. A tabela é 
indexada por dois objetos não colunares: o objeto "snmpEventIndex" identifica o snmpEventEntry que, quando engatilhado, gera uma notificação predefinida pela tupla; e o objeto "partyidentity", que identifica a entidade par de destino para a qual a notificação será enviada.

Dois objetos escalares são associados com esta tabela. $O$ objeto snmpEventNotifyMinInterval define um intervalo mínimo que a entidade SNMPv2 espera antes de retransmitir um InformRequest PDU. Já o objeto snmpEventNotifyMaxRetransmissions define o número máximo de vezes que a entidade SNMPv2 poderá retransmitir um InformRequest PDU.

\subsubsection{SEGURANÇA}

O protocolo SNMP possui uma grande deficiência do ponto de vista de segurança. O SNMPv2 elimina estas deficiências através da implementação de mecanismos adequados. Faremos aqui uma breve abordagem sobre as novas características relacionadas a segurança que foram incorporadas ao protocolo.

O SNMPv2 inclui o conceito de visão de MIB. Esta é definida em termos de uma coleção de subárvores, onde cada subárvore pode ser incluída ou excluída da Visão.

Foi estabelecido o conceito de contexto. Um contexto SNMPv2 nada mais é do que uma coleção de objetos gerenciados por uma entidade SNMPv2, que podem ser alcançados local ou remotamente.

Um Contexto Local é identificado como uma visão de MIB e pode ser visualizado mediante os mecanismos de acesso local. Um Contexto Remoto é identificado como um relacionamento de procuração, onde a entidade SNMPv2 desempenha o papel de um agente proxy de modo a obter acesso às informações de gerenciamento identificadas pelo contexto SNMPv2.

Os contextos SNMPv2 cumprem as funções de relacionar o controle de acesso às visões de MIBs. Tanto no inter-relacionamento entre entidades SNMPv2 gerentes e agentes, quanto entre entidades gerente e agente proxy, os privilégios de controle de acesso selecionado é especificado pelo contexto associado.

Com o uso de contextos, uma abordagem de controle de acesso pode ser estabelecida por quatro elementos: 
1. Alvo - uma entidade SNMP efetuando as operações de gerenciamento requisitadas por uma entidade sujeito;

2. Sujeito - uma entidade SNMP originando requisições de operações de gerenciamento para serem atendidas por uma entidade alvo;

3. Recursos - as informações de gerenciamento nas quais as operações de gerenciamento podem ser efetuadas, expressadas como uma visão local de MIB ou um relacionamento proxy;

4. Privilégios - as operações permitidas, definidas em termo dos PDUs pertencentes a um determinado contexto.

Neste caso, a política de controle de acesso pode ser definidos por três parâmetros. Uma entidade sujeito requisita uma operação de gerenciamento de uma entidade alvo e identifica o contexto da requisição. $O$ contexto pode especificar uma visão de MIB local ou uma entidade remota, atendida por procuração. Para um dado par de entidades sujeito/alvo, podem haver uma política múltipla de controle de acesso, um para cada contexto. O contexto é comunicado por uma entidade sujeito a uma entidade alvo através do cabeçalho de mensagem SNMPv2.

O valor dos parâmetros de privilégio representam a lista de PDUs SNMPv2 que podem ser enviados da entidade sujeito à entidade alvo. O parâmetro é codificado, para cada PDU, mediante a associação de um valor inteiro que seja potência de dois. Isto é:

$\begin{array}{llll}\text { Get } & 1 & -------- & 16 \\ \text { GetNext } & 2 & \text { Get-Bulk } & 32 \\ \text { Response } & 4 & \text { Inform } & 64 \\ \text { Set } & 8 & \text { SNMPv2-Trap } & 128\end{array}$


As principais características de segurança adicionadas ao SNMPv2 estão relacionadas aos serviços de integridade, autenticação e confiabilidade da informação.

- Integridade dos Dados: de forma a assegurar que a mensagem original não venha a sofrer alteração ou perda de conteúdo, provocados por influência de entidades externas, o SNMPv2 utiliza um mecanismo de assinatura digital [Da93] para as mensagens. Desta forma, um algoritmo fica responsável por calcular uma assinatura de 128-bits sobre uma porção apropriada da mensagem SNMPv2. Esta assinatura é então incluída com a mensagem para assegurar que não haja modificações. Além disto, a entidade SNMPv2 que recebe a mensagem se utilizar dos timestamps incluídos pelo remetente para verificar se a mensagem é recente e, consequentemente, passível de ser processada.

- Sigilo dos Dados: Com o propósito de assegurar que os dados não sejam acessíveis às entidades externas não autorizadas, uma porção apropriada de uma mensagem SNMPv2 é codificada usando um algoritmo de criptografia simétrico. O SNMPv2 utiliza o algoritmo DES (Data-Encryption Standard).

- Autenticação: Tem o propósito de assegurar que as mensagens recebidas foram realmente enviadas por entidades autorizadas, e para isso aproveitase do mecanismo de assinatura empregado pelo serviço de integridade dos dados e do mecanismo de sigilo de informação. A assinatura de uma mensagem é calculada sobre uma porção apropriada da mensagem SNMPv2, contendo os endereços do destinatário e do remetente e um par de valores prefixados (timestamps). A seguir estas informações são codificadas utilizando-se uma chave secreta de conhecimento comum entre as partes envolvidas e enviadas ao destinatário. Estes valores podem então ser verificados pelo destinatário, computando-se novamente a assinatura, para fins de autenticação. 


\section{Tecnologia do Gerenciamento}

\subsection{Ambiente de Gerenciamento}

O gerenciamento é freqüentemente visto como uma atividade de cunho única e exclusivamente técnica. Entretanto a responsabilidade final de gerenciamento recai sobre as pessoas que tomam parte no ambiente de redes, e não sobre as máquinas que o integram. Desta forma, Joseph e Muralidhar [Jo88] nos mostra que o ambiente integrado de gerenciamento dentro do qual reside o gerenciamento de redes é uma combinação dos recursos humanos, sociais, e tecnológicos.

A Figura 3.1 nos dá uma visão dos principais componentes que integram o ambiente de gerenciamento, tais como os usuários da rede, os recursos do sistema, a política de gerenciamento e o gerenciamento de rede propriamente dito. Uma descrição detalhada de cada um destes elementos é apresenta em [Jo90].

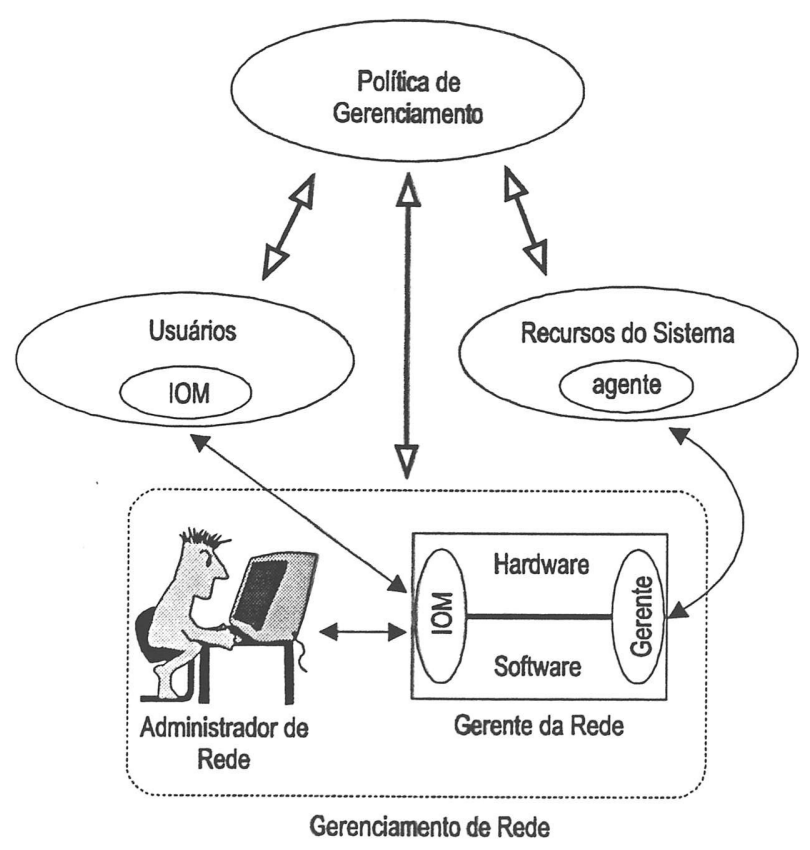

Figura 3.1 - Ambiente de Gerenciamento de Redes 


\subsection{Requisitos de Gerenciamento}

Os ambientes corporativos encontrados atualmente diferem em muito daqueles presenciados em épocas passadas. Para suportar as transições e inovações nestes cenários fez-se necessário o surgimento de novas abordagens organizacionais e tecnológicas.

Se por um lado a absorção destas tecnologias levou a um melhor acomodamento do ambiente, por outro trouxe desarranjos de ordem gerencial que somente puderam ser compensados mediante o emprego de uma sistemática bem elaborada de reestruturação. Esta sistemática está diretamente relacionada aos requisitos de gerenciamento impostos por tais transformações.

Em planos gerais, podemos tratar a "rede" como uma entidade complexa que pode ser composta por uma grande diversidade de recursos de comunicação e serviços. Além disto, apesar de uma rede de computadores poder utilizar uma classe simples de protocolos para a sua completa operação, muitas vezes é necessário o emprego de diferentes classes, a fim de comportar estruturas de redes demasiadamente grandes e complexas.

Neste contexto, os requisitos gerais para o gerenciamento de uma rede de computadores são:

- Habilidade de gerenciar todas as sub-redes de uma rede global, independentemente da classe de protocolos utilizados;

- Habilidade de gerenciar uma ampla gama de recursos de rede. Desde dispositivos de baixo nível (circuitos comutadores, repetidores e modems), até sistemas intermediários (pontes, "roteadores" e conversores de protocolos) entre outros.

- Habilidade de prover um conjunto de funções básicas de gerenciamento, tais como as definidas pelo gerenciamento OSI. 
É bem verdade que temos aqui apenas os requisitos técnicos necessários ao gerenciamento dos recursos de comunicação. Entretanto, como vimos anteriormente, uma solução puramente técnica é insuficiente nos ambiente atuais. Sendo assim, podemos adicionar os seguintes requisitos extras:

- As ferramentas de gerenciamento devem gerar relatórios que sejam de fácil entendimento;

- As funções das ferramentas de gerenciamento devem ser de fácil assimilação e utilização;

- As ferramentas de gerenciamento devem proporcionar uma sensível diminuição da carga de trabalho sobre o administrador da rede;

- Onde possível, as ferramentas devem incorporar os conhecimentos de experientes administradores de redes.

\subsection{Gerenciamento Integrado}

O propósito principal do gerenciamento integrado de redes é fornecer um conjunto de ferramentas que permita gerenciar de forma eficiente todos os recursos de uma dada rede ou conjunto de sub-redes. Segundo Joseph [Jo90], uma integração total do gerenciamento deve tratar quatro aspectos principais:

1. Tanto a interface do usuário quanto os comandos devem ser consistentes para todo o sistema de gerenciamento;

2. As funções de gerenciamento devem empregar conhecimentos e procedimentos comuns ao trabalho efetuado;

3. Os objetos gerenciados pelo sistema devem estar de acordo com um conjunto de definições comuns e consistentes;

4. Os métodos para comunicação tanto entre os objetos gerenciados e os sistemas de gerenciamento quanto entre os próprios sistemas de gerenciamento, devem ser especificados e consistentes. 
O gerenciamento integrado pode resolver muitos dos problemas existentes nas redes da atualidade, como por exemplo, reduzir a carga de trabalho necessária para se administrar a vasta diversidade de dispositivos encontrados.

Como resultado final, o gerenciamento integrado de redes pode reduzir os níveis de conhecimento dos administradores de redes, reduzir os erros no gerenciamento de múltiplas redes e incrementar expressivamente a flexibilidade do gerenciamento.

\subsection{Ferramentas Comerciais}

Muitas ferramentas de auxílio ao gerenciamento foram desenvolvidas e podem ser encontram no mercado. Grande parte foi elaborada pelos próprios fabricantes de dispositivos de conexão de redes com o propósito de permitir o gerenciamento de seus recursos. Porém, poucas destas efetivamente tratam o gerenciamento de uma maneira abrangente e com uma abordagem integrada, ou seja, uma grande parcela destes produtos não atendem os requisitos de gerenciamento apresentados.

Por outro lado, existem produtos comerciais que integram todo um conjunto de ferramentas de gerenciamento e que prestam grande auxilio às funções de gerenciamento encontradas em um ambiente de rede de computadores. De maneira prática, apresentaremos os dois principais produtos comerciais que estão voltados a esta finalidade. São eles o SunNet Manager e o IBM SystemView/6000.

\subsubsection{SunNet Manager}

O SunNet Manager é a plataforma de gerenciamento desenvolvida pela Sun MicroSystems, cuja arquitetura baseia-se no modelo Internet-standard Network Management Framework descrita anteriormente.

Sob o contexto do SunNet Manager existem dois tipos de agentes: um que faz acesso direto aos objetos gerenciados, tais como os dispositivos de armazenamento de massa (Discos rígidos, CD-ROMs, Fitas DAT), Memórias voláteis, Impressoras, Interfaces de rede entre outros; e outro que faz acesso indireto aos objetos gerenciados, através de um agente SNMP (um agente proxy).

O processo de comunicação e troca de informações entre os agentes e o gerente SunNet Manager é efetuado mediante a utilização de uma biblioteca de serviços de 
comunicação e gerenciamento. Os processos gerente e agentes fazem acesso a estes serviços através de uma interface de aplicação (API), implementada via o mecanismo Remote Procedure Call (RPC/XDR) [Su88].

A interface de usuário do gerente SunNet Manager é implementada seguindo o padrão OpenLook GUI. Nesta interface estão dispostos ícones e áreas de controle que possibilitam o acesso às principais funções disponíveis pelo ambiente. Toda a gama de informação é apresentada de forma clara e intuitiva, mediante a utilização de gráficos e textos intercalados. A interação com o administrador de redes é efetuada através do apontador, controlado pelo mouse, e diretamente pelo teclado.

O SunNet Manager possui ferramentas que possibilitam gerar saídas em modo texto planar e gráficos comparativos bi e tridimensionais de dados gerados dinamicamente ou armazenados em arquivos delog.

\subsubsection{IBM SystemView/6000}

O SystemView/6000 é um sistema integrado de gerenciamento de redes voltado a ambientes que se utilizam da classe Internet de protocolos (TCP/IP). Este sistema foi desenvolvido pela IBM e segue de perto as tendências do modelo Internetstandard Network Management Framework.

Como pode ser observado na Figura 3.2, a comunicação entre o gerente e os agentes é efetuada através do protocolo de gerenciamento de redes SNMP (há um esforço por parte da IBM em incorporar o SNMPv2).

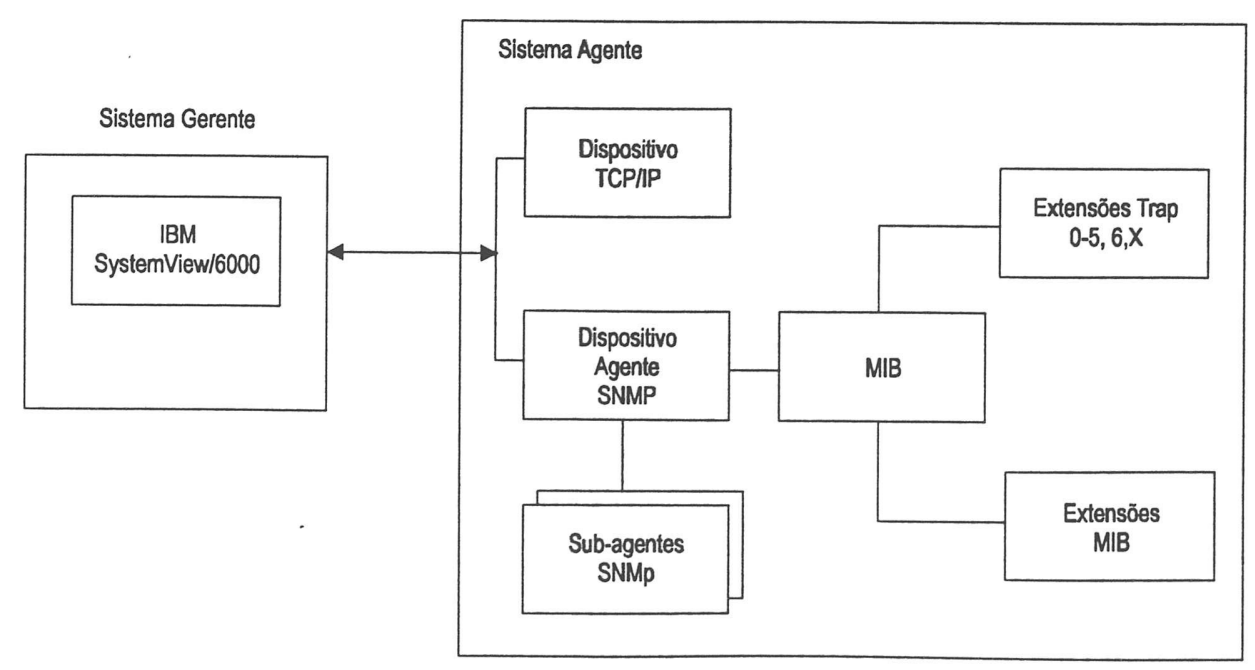

Figura 3.2 - Interação agente-gerente no SystemView/6000 
A interface com o usuário baseia-se no ambiente gráfico padrão OSF/Motif, que também se emprega a abordagem de gráficos, ícones e caixas de diálogos para a troca de informações com o administrador da rede.

O IBM SystemView/6000 apresenta ferramentas de finalidade múltiplas, que permitem que sejam efetuados testes de "conectividade" de redes, descoberta de rotas e extração de informações dos agentes SNMP remotos. As informações a respeito da desempenho global e pontual da rede é apresentada em forma de gráficos de barras. Assim como o SunNet Manager, o IBM SystemView/6000 implementa todo o conjunto de operações do SNMP.

\subsection{MultView}

O Ambiente Integrado de Gerenciamento de Redes MultView leva em consideração, a princípio, o protocolo de gerenciamento SNMP, de forma que os elementos principais que o compõem estão relacionados ao modelo Internetstandard Network Management Framework. O gerenciador constitui-se de cinco módulos principais [Od94] integrados como mostrado na Figura 3.3

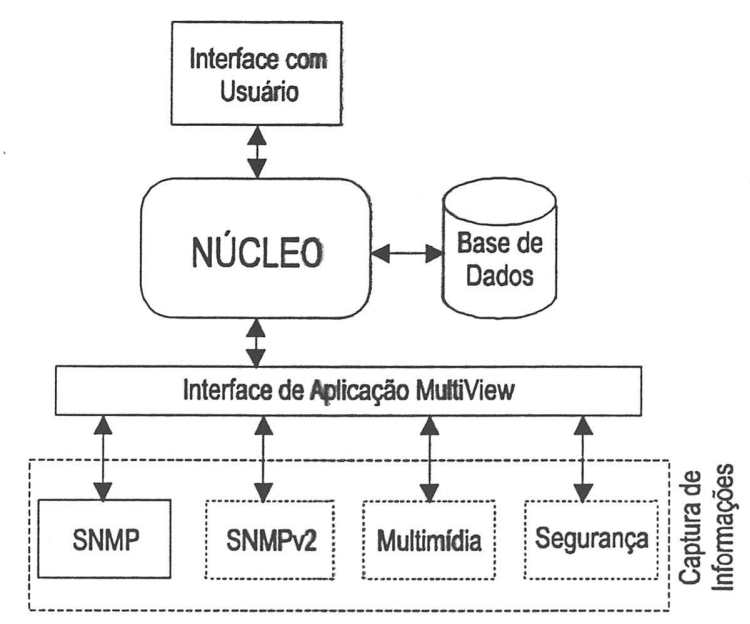

Figura 3.3 - Arquitetura do MultView 


\subsubsection{Módulo de Interface com o Usuário}

O módulo de interface com o usuário contém basicamente as rotinas da interface gráfica, a qual permite a interação com o administrador da rede. Este módulo foi implementado seguindo o padrão gráfico X11R5 OpenView.

As informações são apresentadas graficamente através de janelas, caixas de diálogos, menus e botões, sendo que níveis maiores de detalhes podem ser conseguidos mediante a escolha das funções disponíveis apropriadas.

Gráficos estatísticos mostrando a carga de operação nas sub-redes e estações, indicam possíveis gargalos. Informações valiosas sobre as rotas entres as sub-redes podem ser observadas e caminhos alternativos podem ser selecionados em caso de algum problema com a rota principal.

Antecipando uma tendência tecnológica irreversível, a integração de recursos de Multimídia [Li95] permitirá que imagens e sons digitais sejam mostrados, possibilitando uma integração mais ampla entre os administradores dos vários subdomínios que compõem uma rede. Desta forma, será possível uma troca mais completa e consistente de informações.

\subsubsection{Banco de Dados}

Basicamente, a base de dados permite o armazenamento de três tipos de informações: imagens, sons e textos. Estas informações são obtidas através dos protocolos que integram a classe Internet e que incluem entre outros, o TCP/IP, o ICMP, UDP e os próprios protocolos de gerenciamento SNMP e SNMPv2. Uma outra fonte de informações são os recursos locais onde o processo gerente esta sendo executado (estação de trabalho gerente).

Tais informações podem ser consumidas imediatamente, dependendo única e exclusivamente de como esta será usada, ou armazenada em uma base de dados local, ficando disponíveis para uso futuro. As informações em formato de som e imagem são processadas de forma análoga às informações em formato texto. 


\subsubsection{Módulo Núcleo}

O módulo núcleo compõe-se internamente dos seguintes submódulos:

- Módulo Estatístico: faz o levantamento, avaliação e a apresentação dos dados estatísticos;

- Módulo Gerenciador de Banco de Dados: gerencia e organiza o acesso às informações na base de dados.

- Controle Central: organiza os dados necessários à apresentação gráfica da rede, armazenando-os em uma estrutura adequada; organiza e efetua a busca das informações de gerenciamento em agentes remotos; organiza o fluxo de informações entre o módulo Interface com o Usuário, Banco de Dados e captura de informações.

- Aplicação de Gerência: faz as requisições dos dados de gerenciamento que são obtidos via protocolos de gerenciamento ou localmente.

\subsubsection{Módulo de Captura de Informações}

Sob este módulo encontram-se os demais submódulos que, de uma forma ou de outra, são responsáveis por obterem as informações para o sistema MultView. As operações do protocolo SNMP são implementadas no módulo SNMP, através do qual é possível a comunicação do sistema gerente com os agentes eproxies.

Como parte do Projeto MultView, estão previstas modificações do código existente, de forma a integrar as novas tecnologias emergentes ao escopo do ambiente de gerenciamento. Dentre as modificações estão inclusas:

- Integração e aperfeiçoamento do módulo Multimídia que será responsável por permitir a conexão do gerente com outros gerentes ou equipamentos para passagem de som e imagem;

- Implementação e integração do módulo de suporte ao protocolo SNMPv2, permitindo assim o gerenciamento de uma nova gama de dispositivos e sistemas que serão lançados pelas empresas do setor; 
- Inclusão de um módulo de segurança que possibilitará o gerenciamento de toda a política de segurança da rede sob monitoramento, permitindo inclusive a detecção de tentativas de acessos não autorizados aos recursos do ambiente e a tomada de decisões de contramedida.

Todos estes adendos tecnológicos encontram-se em fase de desenvolvimento pelo Grupo de Multimídia em Redes do Laboratório Intermídia, como parte do programa de mestrado e doutorado do Instituto de Ciências Matemática de São Carlos, aliados a outros institutos e instituições de fomento à pesquisa (CAPES, CNpQ, FAPESP e IBM).

\subsubsection{Interface de Aplicação MultView}

Um dos objetivos buscado na elaboração do gerenciador é a flexibilidade do sistema de forma que os módulos possam ser inseridos ou removidos sem que sejam necessárias grandes modificações no módulo Núcleo. Para tal, esta interface de aplicação fornece um ponto de acesso entre o núcleo e os módulos de captura de informação, de forma a preservar a independência física destes, sem atrapalhar a forte dependência lógica. 


\section{Uma Nova Arquitetura para o MultView}

\subsection{Aspectos evolutivos}

Sem dúvida, a inovação e a evolução tecnológica refletem as necessidades organizacionais e estruturais dos ambientes atuais. A velocidade com que tais transformações vêm ocorrendo surpreende mesmo as organizações que contribuem diretamente com a escalada tecnológica, deixando-as constantemente sujeitas a centrarem esforços no sentido de manterem atualizados seus produtos.

Dentro desta perspectiva evolutiva, a ferramenta MultView foi submetida a uma criteriosa avaliação de sua arquitetura e estrutura funcional, buscando identificar os principais problemas advindos da desatualização tecnológica a que ficou exposta. Como resultado, foram estabelecidas novas diretrizes de desenvolvimento, centrada na busca de uma arquitetura tecnologicamente superior e capacidade de gerenciamento flexível, sob demanda.

Neste contexto, foram definidos os requisitos básicos e essenciais a serem seguidos. Tais subsídios foram elaborados mediante uma extensiva prospecção tecnológica, tendo como critério fundamental a adesão às especificações de padrões abertos internacionais, como os propostos pelas organizações ISO (International Standard Organization), ITU (International Telecommunication Union), OMG (Object Management Group) IAB (Internet Architecture Board) e W3C (World Wide Web Consortium).

Outra importante fonte de informação para a concepção de tais requisitos vieram da avaliação de produtos em áreas de tecnologias afins, tais como Jigsaw (W3C, MIT, INRIA), Netscape Server's Architecture (Netscape, Inc) e Jeeves (Sun Microsystems). 


\subsection{Requisitos}

Centrado nas premissas de uma arquitetura tecnologicamente superior e capacidade de atualização facilitada, foram elaborados os seguintes requisitos:

- Uma ferramenta de gerenciamento deve ser facilmente portável para diferentes plataformas de sistemas operacionais e arquiteturas de hardware, exigindo-se um mínimo de esforço neste sentido.

- Uma ferramenta de gerenciamento deve ser amplamente extensível em relação às suas capacidades, possibilitando a inserção de novas funcionalidades sem a necessidade de re-codificações das partes já existentes. Além do mais, deve apresentar como característica desejável a capacidade de extensão dinâmica, sem a necessidade de interrupções de seu funcionamento.

- Uma ferramenta de gerenciamento deve possuir capacidades modernas e eficientes de comunicação com o administrador da rede, provendo-o de total controle sobre os recurso disponíveis, independentemente da sua localização geodésica e do grau de segurança do meio de comunicação utilizado. Com tal intuito, protocolos confiáveis e seguros de comunicação devem ser empregados.

- Uma ferramenta de gerenciamento deve utilizar-se das tecnologias e especificações abertas complacentes, devidamente padronizadas por entidades a contento, e de reconhecida superioridade funcional.

O direcionamento destes requisitos teve como resultado o estabelecimento de uma nova arquitetura, baseada em tecnologias emergentes e que serviu como base para a implementação inicial da nova ferramenta de gerenciamento de redes MultView. Antes, entretanto, são apresentadas as tecnologias utilizadas em sua implementação. 


\subsection{Tecnologias emergentes}

A cada dia, surgem novas e modernas tecnologias que possuem como característica comum a generalização de suas aplicações nas mais variadas áreas de interesse da humanidade. No segmento de Tecnologia da Informação, este comportamento pode ser amplamente observado; diga-se de passagem a integração de áreas como Hipertextos, Protocolos de Comunicação, Criptografia de Dados e Multimídia, que culminou em um dos mais extraordinários sistemas de informação de nossa era. A Interntet e sua malha de navegação World Wide Web. Seguem algumas das tecnologias que integram a arquitetura da ferramenta de gerenciamento de redes MultView.

\subsubsection{Java}

Desenvolvida pela Sun Microsystems, a linguagem de programação Java [Go96] apresenta uma série de características únicas que tornaram-na um marco na história da informática moderna. Tais características incluem e não limitam-se às que se seguem:

- Java é um linguagem fortemente baseada em tipos e que integra todos os conceitos de orientação a objetos [Bo91];

- Resolução de dependências em tempo de execução. Esta característica é de extrema importância, pois permite não apenas a carga dinâmica de classes como também a utilização de referências cruzadas nas definições das mesmas (i.é. classes distintas podem possuir referências entre si).

- Independendência de plataforma. Característica importante da linguagem Java, foi a principal responsável pela rápida e maciça aceitação da mesma. O código gerado pelo compilador (conhecido por bytecode) não é direcionado a nenhuma arquitetura específica de processador, mas sim à especificação de uma máquina virtual conhecida como Java Virtual Machine [Li97].

- Código Transportável. Característica advinda das demais, permite o envio e recebimento de código, possibilitando sua posterior execução após uma completa checagem de segurança. Tais códigos são conhecidos por Java 
Applets e são comumente executados nas máquinas virtuais atreladas aos ambientes de navegação (Browsers).

\subsubsection{CORBA}

O aumento e a rápida proliferação do número de hardwares e softwares disponíveis impulsionaram o desenvolvimento em busca de uma arquitetura que permitisse a interoperabilidade entre dispositivos de rede. Como resposta a esta necessidade, o OMG (Object Management Group) propôs a especificação CORBA (Common Object Request Broker Architecture) [Be95], que de uma forma simples, permite a comunicação entre objetos de aplicações, independente de suas localidades. Junto à CORBA, o OMG também definiu a IDL (Interface Definition Language) e as APIs (Application Programming Interfaces), utilizados para o desenvolvimento de aplicações distribuídas.

O relacionamento entre os objetos distribuídos é realizado através de uma arquitetura cliente-servidor, onde há a presença do ORB (Object Request Broker) como agente intermediário. Através do ORB um cliente pode executar um método de um objeto local ou remoto qualquer. De fato, o ORB intercepta a chamada a um método, localiza o objeto que o implementa, executa o método e por fim retorna os resultados. Tudo isso acontece de forma transparente, sem a necessidade do cliente saber onde o objeto destino encontra-se, a linguagem de programação empregada em sua implementação, o sistema operacional onde reside ou qualquer outro aspecto relacionado à interface com o objeto. Neste cenário, a IDL tem como proposta simplificar o desenvolvimento destas aplicações, pois permite descrever detalhadamente as interfaces dos métodos de cada objeto que compõe uma aplicação distribuída.

\subsubsection{HTTP}

O HyperText Transfer Protocol [Da97] é um protocolo aberto, responsável por permitir o transporte de informações de tipos variados sobre uma rede de arquitetura TCP/IP. Este protocolo é comumente empregado no tráfego de códigos HTML [Fi97], executáveis bytecode Java, multimídia, textos planares ASCII, entre outros. 


\subsubsection{SSL}

O padrão de segurança de informação SSL (Secure Sockets Layer), desenvolvido inicialmente pela Netscape [Fr96], especifica uma camada de segurança entre a aplicação e a porta de comunicação, protegendo o conteúdo da informação mediante sua criptografia. Tal especificação encontra-se atualmente em fase de padronização internacional, devendo emergir como uma alternativa segura para o transporte de informações de caráter restritivo. O protocolo em si é baseado nos principais algoritmos de criptografia de dados e provê suporte à certificação de autenticidade, tanto do servidor quanto do cliente envolvidos na troca das informações.

\subsection{Elementos da Arquitetura}

A pesar da estrutura de gerenciamento Internet mostrar-se desatualizada frente às novas proposta tecnológicas [De96], ainda sim apresenta-se como o principal padrão existente, com funcionalidade comprovada. Sendo assim é evidente a necessidade de sua adoção como elemento de adequação a realidade atual.

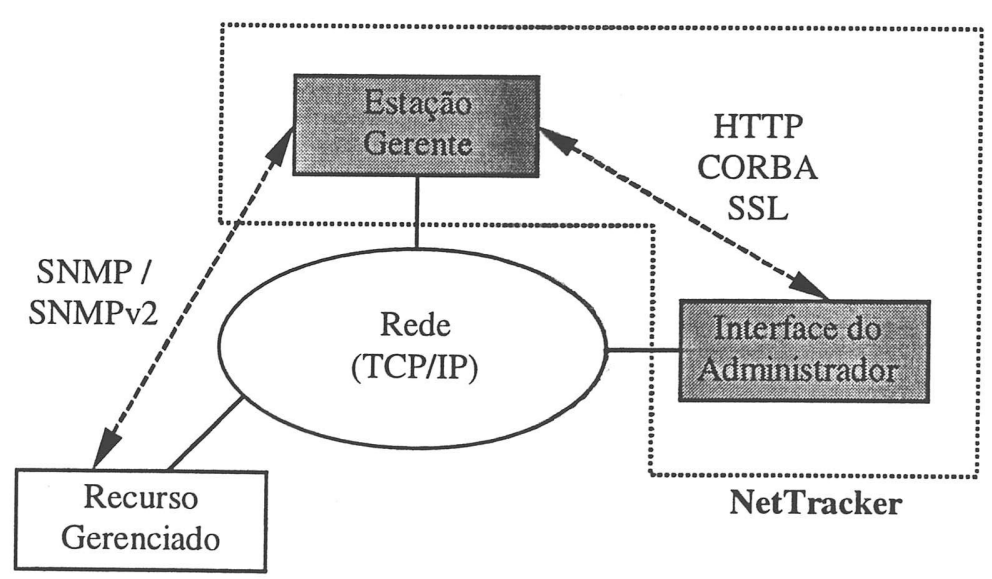

Figura 4.1 - Relacionamento de Comunicação intramódulos

A Figura 4.1 sintetiza o relacionamento entre os elementos da estrutura de gerenciamento que integram o ambiente gerenciado pela ferramenta MultView, e que seguem de perto a estrutura do gerenciamento Internet. Tais elementos são descritos a seguir. 
- RECURSO GERENCIADO - representa todos os elementos que compõem a rede e que sejam providos de capacidades de gerenciamento Internet (i.é, Routers, Bridges, Hosts, etc), comunicando-se diretamente com a estação de gerenciamento, via protocolo SNMP ou SNMPv2.

- ESTAÇÃO GERENTE - parte fundamental da ferramenta integrada MultView, este elemento atua como agente centralizador das informações de gerenciamento, ficando responsável por prover todas as funções de controle e monitoração ao administrador.

- INTERFACE DO ADMINISTRADOR - é o elemento responsável por permitir a interação entre a ferramenta MultView e o administrador da rede. É importante notar que esta cumpre não apenas o papel de agente difusor de informação, mas também o papel de elemento descentralizador de gerenciamento

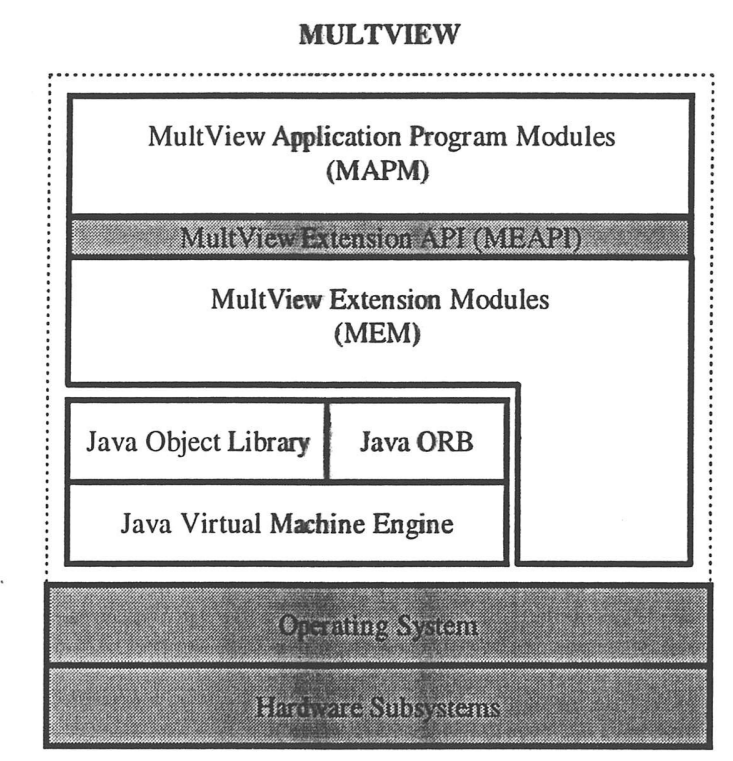

Figura 4.2 - Arquitetura do Sistema MultView

Sob a ótica de gerenciamento, a Figura 4.2 apresenta uma visão de alto nível da arquitetura do novo MultView, que é composta por cinco camadas interdependentes: Módulos de Programas de Aplicação (MAPM), Interface de Extensão de Aplicações (MAPI), Módulos de Extensão (MEM), Biblioteca de Objetos Java e Máquina Virtual Java. A seguir são descritos os papeis funcionais de cada uma destas camadas. 


\subsubsection{Camada de Módulos de Programas de Aplicação}

A camada MAPM trata dos aspectos funcionais agregados ao sistema MultView, incorporando as aplicações responsáveis por proverem as funções de gerenciamento e de comunicação com o administrador. Como exemplos de elementos componentes desta camada, pode ser citada a aplicação exemplo (Filtro de Traps), juntamente com as demais aplicações de gerenciamento.

\subsubsection{Camada de Interface de Extensão de Aplicações}

Nesta camada, encontram-se disponíveis as interfaces de programação que fornecem acesso às funções implementadas pelos Módulos de Extensão. No contexto da tecnologia de objetos, que é amplamente empregado neste projeto, esta camada é composta por todas as interfaces dos objetos codificados na camada MEM.

\subsubsection{Camada de Módulos de Extensão}

Todos os objetos responsáveis por proverem funções básicas de apoio às funções de gerenciamento serão integrados à esta camada. Tais funções desempenham o papel de alicerce para os programas de aplicações que se encontram na camada superior. Basicamente, esta é composta pelos seguintes sub-módulos:

- HTTPServer - Permite ao MultView disponibilizar serviços de configuração remota através de requisições HTTP. Este módulo possibilita que as interfaces de usuário possam ser transparentemente enviadas ao administrador, independentemente de sua localidade no ambiente.

- Database - Este módulo é responsável por prover, de forma tranparente, acesso à mais variada gama de SGBDs disponíveis no mercado. Para tal prevêse a utilização do protocolo JDBC (Java Database Connection) ou ainda de drivers nativos oferecidos pelos fornecedores dos produtos.

- SNMP - todas os recursos de gerenciamento presentes no protocolo SNMP estão agrupados neste módulo. inicialmente, foram agregadas as funcionalidades da implementação de domínio público Advent SNMP Java API [Ad96]. Prevê-se, contudo, a mobilização de esforços no sentido de implementá-lo no futuro. 
- SNMPv2 - com propósito semelhante ao anterior, este módulo disponibilizará as funções de gerenciamento da nova versão do SNMP (SNMPv2). Existe um trabalho paralelo, de implementação deste módulo, sendo efetuado como parte do programa de mestrado do ICMSC.

- Security - este módulo tem o objetivo de disponibilizar todas as funções que tratem dos aspectos de segurança da ferramenta MultView (i. é, SSL, MD5, DES, RSA, entre outros). Além do mais, existe um projeto, do programa de doutorado do IFSC, que visa incorporar funcionalidades extras de segurança ao MultView e que serão integradas posteriormente a este módulo.

\subsubsection{Biblioteca de objetos Java \& Java ORB}

Nesta camada encontram-se todos os objetos que compõem as classes da linguagem Java, e que são responsáveis por permitirem a implementação de grande parte das camadas superiores. Além disto, como previsto para implementações futuras da linguagem Java, serão incorporados pacotes de funções de comunicação e controle de objetos remotos, que seguem as especificações CORBA. Já encontra-se disponível uma implementaçãoalpha desta biblioteca.

\subsubsection{Maquina Virtual Java}

Sem dúvida, é o coração da nova arquitetura do MultView. Sobre ela estão assentadas todas as principais características inovadoras da ferramenta: carga e descarga dinâmica de módulos, independência de plataforma, linkedição dinâmica e em tempo de execução de classes, acesso remoto a objetos, códigos binários transportáveis, etc. 


\section{Detalhes Operacionais}

Aderindo integralmente à nova arquitetura e paradigma propostos, o MultView foi remodelado para esta nova realidade. Para um melhor entendimento do mesmo, é necessário definir alguns dos conceitos básicos, porém essenciais, adotados ao logo do projeto. Estes conceitos englobam a estrutura e relacionamento dos objetos no sistema, além da definição e operação das aplicações MultView.

\subsection{A estrutura e o relacionamento dos objetos no sistema.}

O novo MultView é composto por duas categorias principais de objetos: Objetos de Sistema e Objetos de Aplicação. Os primeiros definem os objetos que integram o ambiente básico de execução do sistema, efetuado a carga dinâmica e posterior ativação dos Objetos de Aplicação. Estes últimos, por sua vez, definem de fato as aplicações do sistema MultView. A Figura 5.1 dá uma visão mais detalhada desta estrutura organizacional.

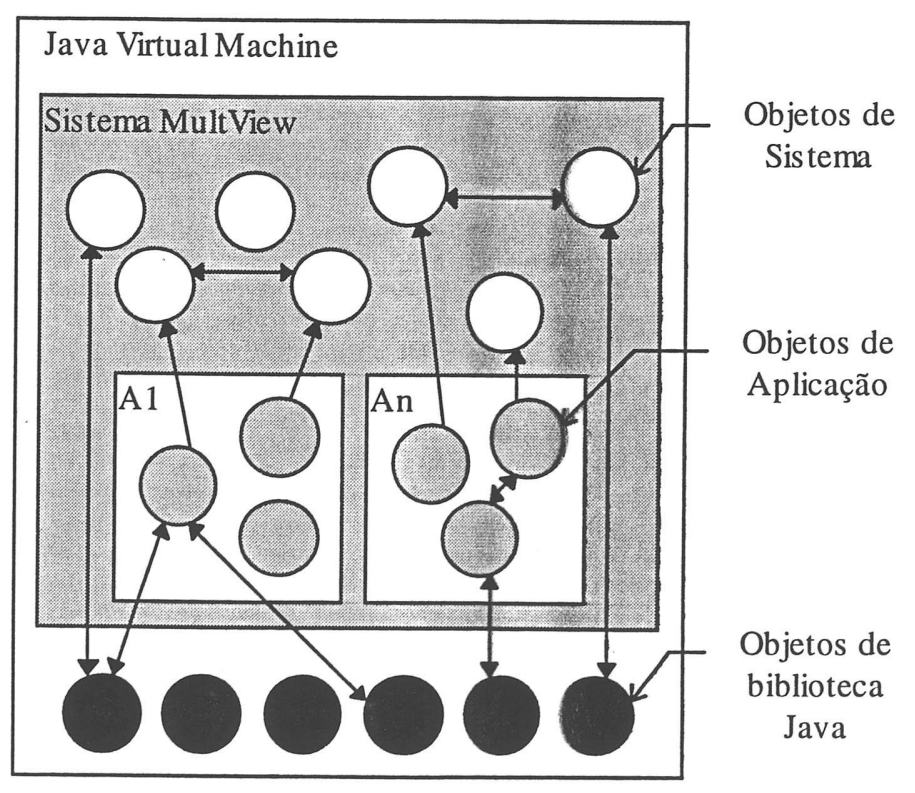

Figura 5.1 - Estruturação dos Objetos MultView 
Obedecendo a esta estrutura de relacionamento, o processo de ativação e execução do sistema MulView cumpre os seguintes passos:

1. Após o processo de ativação da Máquina Virtual Java, a classe principal do sistema (MultView) é carregada, checadas suas dependências e posteriormente ativada. Procedimento este comumente aplicado às aplicações Java;

2. Após ser instanciado pela etapa anterior, e de posse do controle executivo, o método construtor configura o sistema, criando as estruturas de suporte e controle necessárias. Neste processo são instanciados e ativados os objetos ditos essenciais, tais como MVHttpServer, MVManager, MVLoader. O primeiro funciona como um servidor HTTP e fica responsável por permitir o interfaceamento do sistema MultView com o administrador da rede, através de um Browser Web qualquer. Já o segundo é responsável por controlar a criação e o gerenciamento dos objetos MultView (MVAplication) que são instanciados a partir de classes carregadas dinamicamente pelo ambiente. $O$ objeto MVLoader é tratado logo a seguir. Ao término desta etapa, todos os objetos de sistema estão estabelecidos e em processo de execução;

3. Ativado como uma thread Java, o objeto MVLoader inicia o procedimento de carga e registro de todos os objetos de aplicação. Para tal é examinado o arquivo de cadastro de aplicações (MVAppReg.cfg), buscando e efetuando a carga dinâmica de cada um dos módulos das aplicações cadastradas. Em princípio, estão cadastradas as aplicações de suporte ao sistema (ManagerSNMP, Logger, Trigger, Database). A aplicação de Filtragem de Traps (Trapper) é também carregada nesta etapa;

4. Após a carga e durante o processo de ativação dos módulos de aplicação, mencionados anteriormente, cada qual efetua o registro de seus atributos em um objeto de registro de atributos (MVApplicationAttributes). Isto é necessário, pois através deste registro é possível o controle e monitoração de cada atributo pelo gerenciador de atributos do sistema (MVManager).

Ao término deste processo de ativação, o sistema está plenamente operacional e apto a interagir com o administrador da rede. 


\subsection{Definição e operação das aplicações MultView}

Cada aplicação ou módulo de aplicação que pode ser integrado ao sistema é composto por um conjunto de definições de objetos que interagem entre si para proporcionar a funcionalidade necessária e/ou esperada. Para tal, um destes objetos (objeto de ativação) deve ser definido obedecendo a duas regras essenciais, apresentadas a seguir.

- A definição (Classe) de um objeto principal de aplicação deve necessariamente herdar os atributos e métodos da superclasse MVApplication, como mostrado na Figura 5.2. Esta última, por sua vez, define os métodos abstratos de interfaceamento dos objetos do sistema, apresentados anteriormente, com os objetos de aplicação;

- Todo objeto de ativação deve implementar obrigatoriamente os métodos run e perform, mostrados na Figura. 5.3. O primeiro deve codificar as instruções e operações que estabelecem, de fato, a aplicação no ambiente operacional do sistema, e será invocado logo após sua carga pelo mesmo. O último deve conter código de manuseio de solicitações HTTP.

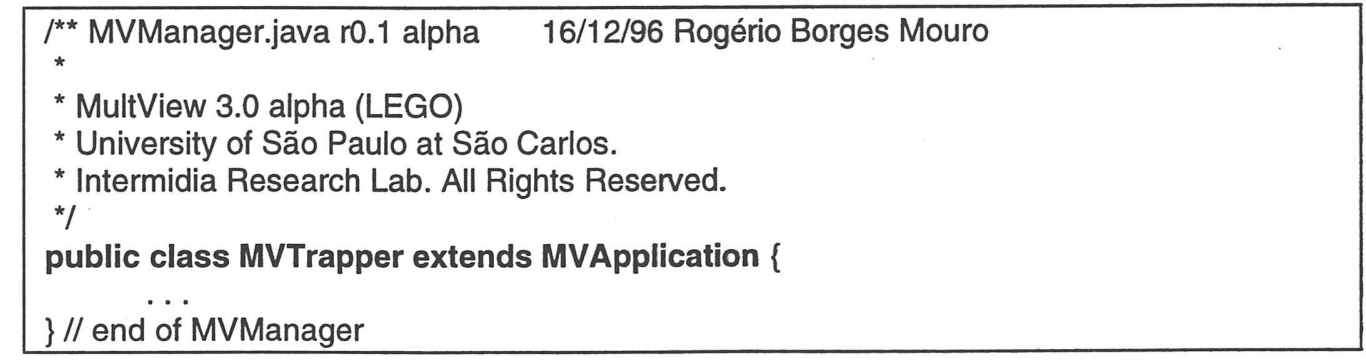

Figura 5.2 - Definição de uma aplicação MultView

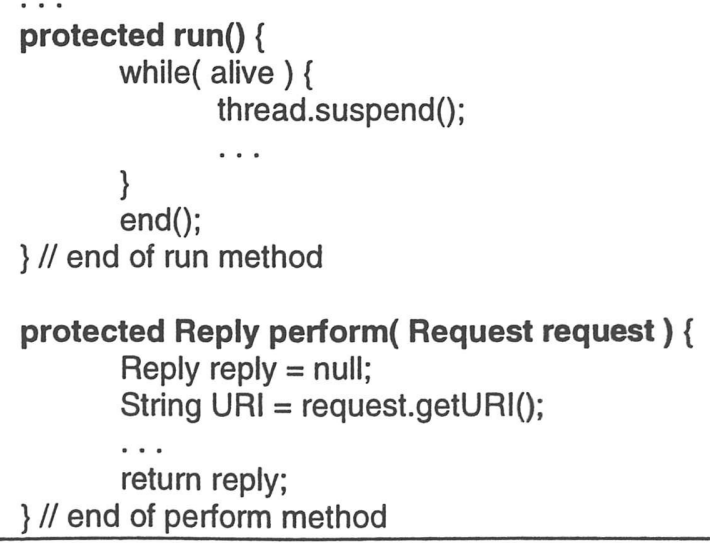

Figura 5.3 - Funções essenciais 
Além da obrigatoriedade do cumprimento destas regras, e caso haja necessidade de definir atributos de controle para a aplicação, cada um destes atributos devem ser definidos utilizando-se os objetos preestabelecidos para este propósito (MVInt, MVString, MVBoolean e MVByte). É importante ainda salientar que cada atributo deverá registrar-se explicitamente, através do método registerAttribute, no objeto de atributos (MVApplicationAttributes) da aplicação do qual fazem parte.

Seguindo estas regras na definição de uma aplicação, seu relacionamento com outros objetos no sistema se dá de forma transparente, mesmo quando as classes alvo do relacionamento ainda não estão carregadas e disponíveis. Neste caso o próprio ambiente Java se incumbe de resolver tais dependências, carregando-as e ativando-as quando necessário.

\subsection{Aplicações de Suporte ao Sistema}

As aplicações de suporte ao sistema são aquelas carregadas durante o processo de inicialização do MultView, como já descrito anteriormente. São elas: ManagerSNMP, Logger, Trigger e Database

\subsubsection{ManagerSNMP}

A aplicação ManagerSNMP é responsável por gerenciar as informações de gerenciamento SNMP contidas em MIBs (Management Information Base) controladas e mantidas por agentes SNMP. O processo de obtenção das informações é realizado de duas formas: polling e trapping. No pooling, a aplicação gerente faz requisições periódicas aos clientes; no trapping, mensagens são enviadas ao gerente notificando-o sobre a ocorrência de eventos anormais ou prédeterminados, sem que estas tenham sido requisitadas pelo gerente.

\subsubsection{Logger}

A função do Logger é gerenciar os arquivos de log das aplicações do sistema MultiView. As aplicações devem referenciar o objeto Logger para enviar as mensagens formatadas com três campos: type (determina o tipo da mensagem), priority (define a prioridade de tratamento da mensagem) e message (a mensagem 
que será armazenada no $\log$ ). A determinação em qual arquivo de log será armazenada a mensagem é de responsabilidade do administrador, que através de uma interface HTTP (via método perfom()) determina qual arquivo está relacionado ao par (type, priority).

\subsubsection{Trigger}

O Trigger é responsável por executar funções ou tarefas que sejam dependentes de algum evento que possa ocorrer no ambiente. Estas funções são configuradas seguindo um relacionamento com algum evento determinado, por exemplo: o recebimento de uma notificação da perda de comunicação entre um par de roteadores, pelo módulo de filtragem de traps, poderia disparar uma tarefa que estabeleceria uma rota alternativa, através da inserção de uma rota extra na tabela de roteamento de um roteador disponível.

\subsubsection{Database}

A Database gerencia as consultas à base de dados realizadas pelas aplicações, executando os comandos SQL e retornando o resultado da consulta. Para ter acesso as funcionalidades do Database, as aplicações referenciam o objeto Database que possui todos os métodos necessários para a criação e manutenção da base. Por exemplo: as informações recebidas e coletadas pelo ManagerSNMP são armazenadas em uma base de dados para que estas estejam disponíveis às outras aplicações, que também fazem acesso às informações através do Database.

\subsubsection{Filtro de Traps}

A aplicação de filtragem de traps (Trapper) é responsável por receber notificações de ocorrências de eventos importantes, que possam vir a ocorrer em um agente SNMP ou SNMPv2, e filtrá-las de modo a apresentar informações baseadas em padrões e critérios pré-definidos pelo administrador da rede. Desta forma pode-se estabelecer ações que possam ser disparadas automaticamente pelo sistema em reação a um estímulo fornecido por um dos filtros. 
Em princípio, o sistema está tratando apenas o protocolo SNMP via SNMP Advent API [Ad96], dado a falta de uma API SNMPv2 disponível. Existe, entretanto, um trabalho desenvolvimento como parte do programa de mestrado do Instituto de Ciências Matemáticas de São Carlos, que visa implementar uma API SNMPv2, a qual poderá ser incluída posteriormente no sistema MultView.

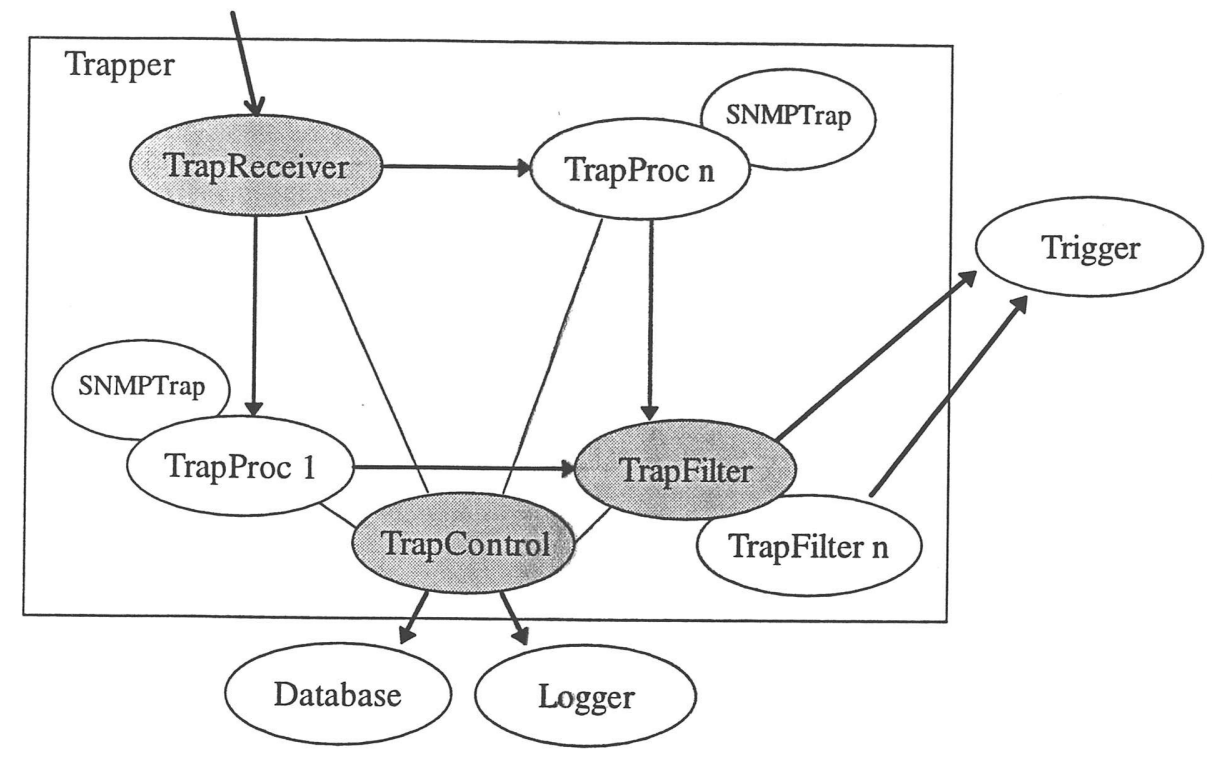

Figura 5.4 - Objetos da Aplicação de Filtro de Traps

A composição de objetos e o interrelacionamento destes, dentro da aplicação de filtragem de eventos, pode ser observado na Figura 5.4. De fato, iniciada através da carga e ativação da classe principal (Trapper), são criados e ativados os objetos TrapReceiver, TrapFilter e TrapManager, cujas funções são descritas a seguir.

\section{TrapReceiver}

Este objeto opera como uma Thread Java de prioridade alta e fica responsável por receber as notificações (Traps) enviadas pelos agentes SNMP da rede. Para tal, é alocado um objeto Soket sobre a porta de notificação de Traps (porta 162). Assim que uma notificação é recebida, é criado um objeto de tratamento de trap (TrapProc), que executa como uma Thread, liberando o TrapReceiver que volta ao estado inicial de espera. 
Este novo objeto de processamento de trap fica, então, responsável pela recepção da notificação e o seu posterior tratamento, gerando como resultado um objeto do tipo SNMPTrap já totalmente certificado e aprovado.

\section{TrapFilter}

É o objeto responsável por analisar o conteúdo de um trap, buscando a ocorrência de padrões e obedecendo os critérios determinados pelo administrador da rede.

No momento inicial da ativação da aplicação de Filtragem de Traps, existe um único filtro ativo. Este é responsável por aceitar prontamente todo e qualquer tipo de notificação enviada pelos agentes SNMP que compõem a estrutura de rede.

Mediante a intervenção do administrador, é possível a criação e ativação de filtros adicionais que obedeçam a uma série de requesitos: Endereço de origem, Comunidade a que pertence, Tipo de Notificação (reinicialização de dispositivo, alteração do status dos enlaces das interfaces, falha de autentificação, perda de comunicação entre um par EGP pré-definido, eventos específicos), tempos mínimo e máximo de operação do agente determinado.

A satisfação dos requisitos de um filtro, por parte de um evento, pode ter como conseqüência o disparo de uma tarefa ou procedimento pré-configurado no módulo de Trigger. Com isto é possível, por exemplo que o recebimento de uma notificação de perda de comunicação entre um par EGP de um determinado gateway, tenha como consequência o estabelecimento de uma rota alternativa, mediante a inserção automática de uma rota extra na tabela de roteamento de um outro gateway disponível.

\section{TrapControl}

Este objeto possui a função de permitir e estabelecer o controle dos demais objetos da aplicação Trapper. Através dele são criados, ativados, desativados, destruidos e configurados os filtros de traps adicionais. Ele também possibilita o registro e controle dos atributos que definem o comportamento dos demais objetos, além de funcionar como iterface de aplicação, pemitindo a consulta, inserção e remoção dos objetos SNMPTrap em uma base de dados. 


\section{Considerações Finais}

\subsection{Conclusões}

A dimensão e abrangência das redes de computadores vêm aumentando gradativamente ao longo dos últimos anos. Este fenômeno possui como reflexo direto o incremento expressivo da complexidade das redes. Em conseqüência disso, abordagens e ferramentas eficientes para o gerenciamento desses ambientes tornam-se necessários e de extrema importância.

Paradoxalmente, o grande inconveniente em se efetuar a adequação das ferramentas já existentes às novas tecnologias é o fato de que tais ferramentas foram concebidas como elementos de vanguarda tecnológica em suas atualidades, impossibilitando ou reduzindo suas possibilidades de atualizações futuras.

Inclusa neste contexto, a ferramenta de gerenciamento de redes MultiView foi remodelada e adequada aos novos avanços tecnológicos. A adoção de uma nova e moderna arquitetura operacional, em conjunto com as propriedades oferecidas pelo ambiente de programação Java, permitiu agregar as funcionalidades de extensão dinâmica de capacidade, independência de plataforma operacional e integração com as tecnologias Internet e Web.

A pesar de ter sido projetada especificamente para atender as necessidades da ferramenta de gerenciamento de redes MultiView, tanto o ambiente quanto a arquitetura mostram-se extremamente versáteis e abertas às mais diversificadas utilizações. Para isto, basta codificar módulos de aplicações independentes dos recursos de gerenciamento, aproveitando a interface de controle $\mathrm{e}$ as possibilidades da API MultiView existentes.

\subsection{Trabalhos Futuros}

De fato, este projeto serviu como base para uma nova, avançada e poderosa ferramenta de gerenciamento de redes de computadores. Entretanto, dada a extensão das possibilidades em torno da mesma, apenas a parte fundamental pôde ser desenvolvida e implementada. Seguem alguns dos melhoramentos que, feitos de forma ordenada e dentro dos critérios preestabelecidos, trarão grandes benefícios e ilimitadas capacidades às futuras versões do MultiView: 
- Mesmo com o intenso movimento ao redor do protocolo de gerenciamento SNMPv2, o SNMP mostra-se como o principal meio de difusão de informações de gerenciamento nos ambientes de rede de computadores da atualidade. Isto acontece pelo fato de que indecisões no processo de especificação têm atrasada a elaboração e adoção de um padrão definitivo. Entretanto, seguindo tendências na área, a incorporação de uma API SNMPv2 trará grandes benefícios ao sistema, visto que será aberto um leque de possibilidades do ponto de vista de segurança do gerenciamento;

- Um dos grandes fatores que permeiam as possibilidades de uma ferramenta de gerenciamento de rede é o de segurança da própria ferramenta. Este aspecto foi vislumbrado, porém pouco explorado, na arquitetura do novo MultiView. Houve a preocupação no sentido de eliminar a possibilidade de implementação e carga de aplicações viróticas no sistema, sem contudo eliminar instabilidades advindas de falha ou erros ocasionados por uso indevido da ferramenta. $O$ estabelecimento de uma política de tratamento destes eventos e sua implementação é de fundamental ajuda rumo a um ambiente estável e potencialmente confiável;

- Os módulos de gerenciamento presentes no versão anterior do MultView agregam grandes funcionalidades ao mesmo. Seria de grande valia a migração de tais módulos para a plataforma atual, principalmente no que diz respeito ao módulo de descoberta automática e apresentação da topologia da rede;

- A interface de controle de aplicações serve como ponto de acesso ao ambiente e todas suas capacidades. Apesar der terem sidos considerados métodos de autenticação e controle de acesso à tal elemento, não foi possível a inserção de tais métodos no protótipo inicial, principalmente devido a indisponibilidade de uma API SSL. Seria de grande valia a inclusão de tais recursos, mediante a adoção de uma futura API. 


\section{Apêndice}

\subsection{A - Primitivas Operacionais SNMPv2}

- SNMPv2 GetRequest PDU - Mesmo se um dos valores das instâncias dos objetos não puder ser processada, ainda sim uma "Response PDU" é gerada pela entidade SNMPv2 Agente e, consequentemente, devolvida à entidade Gerente requisitante.

O processamento das variáveis é efetuado mediante a aplicação das seguintes regras: no caso da inexistência do prefixo de identificação do objeto requerido, o valor do campo de resposta correspondente é preenchido com "noSuchObject"; No caso da inexistência da variável requisitada, o valor do campo correspondente na mensagem de resposta é preenchido com "noSuchInstance"; caso contrário o valor deste campo é preenchido com o valor da variável requisitada.

Se por um motivo qualquer não for possível processar o valor da variável requisitada, então o campo correspondente na mensagem de resposta é deixado em branco. Outro problema que pode ocorrer está relacionado ao limite máximo imposto ao tamanho da mensagem de resposta. Caso este limite seja ultrapassado, o "Response PDU" é descartado e um novo é construído contendo um sinalizador de erro "tooBig" e os campos das variáveis de amarração serão deixados vazios.

- SNMPv2 GetNextRequest PDU - O processamento das variáveis é efetuado mediante a aplicação das seguintes regras: é retornada a próxima variável que esteja em ordem lexicográfica; caso não seja possível determinar a sucessora nesta ordem, o valor do campo correspondente e preenchido com o valor "endOfMibView".

- SNMPv2 GetBulkRequest PDU - Basicamente uma GetBulkRequest inclui uma lista de $(\mathrm{N}+\mathrm{R})$ variáveis. As $\mathrm{N}$ primeiras são processadas de forma análoga a GetNextRequest, obtendo-se os valores sucessivos na ordem lexicográfica. Já para as últimas $\mathrm{R}$ variáveis, serão retornados múltiplos sucessores. 
Para isto, a GetBulkRequest PDU contém dois campos adicionais: "nonrepeaters" e "maxrepetitions", responsáveis sucessivamente por identificar o número de variáveis que deverão ser tratadas normalmente $(\mathrm{N})$ e a quantidade máxima de sucessores lexicográficos retornados para cada variável restante.

No caso de ocorrência de uma falha no processamento de alguma variável, então um Response PDU é gerado, contendo o código de erro "genErr" e o índice da variável em que ocorreu o problema. Outro erro possível seria o fato de que o tamanho da mensagem de resposta extrapolasse os limites máximos definidos. A regra que se aplica neste caso é simplesmente preencher a mensagem de resposta até o limite máximo de seu tamanho.

- SNMPv2 SetRequest PDU - Esta operação é conduzida em duas fases: na primeira, cada variável de amarração é validada e se, e somente se, todas sejam válidas, então seus valores são alterados na fase seguinte. Um tratamento mais elucidativo sobre os processos de validação e atualização é feito por [St93].

- SNMPv2 Trap PDU - O Trap PDU é gerado por uma entidade SNMPv2 que esteja cumprindo o papel de agente, e não gera resposta de confirmação por parte da estação de gerenciamento que a recebe.

- SNMPv2 InformRequest PDU - O endereço de destino para o envio da resposta é especificado na tabela "SNMPv2EventeNotifyTable", definida na MIB de comunicação Gerente-Gerente, ou para o endereço de destino especificado pela aplicação requisitante.

Quando um InformRequest PDU é recebido, a entidade SNMPv2 que o recebe inicialmente determina o tamanho de uma mensagem que encapsule um Response PDU com os mesmos conteúdos do InformRequest PDU. Se este tamanho exceder as capacidades limitantes, um Response PDU é construído contendo o código de erro "tooBig". Caso contrário, a entidade SNMPv2 receptora passa o conteúdo da resposta para uma aplicação de destino e gera um Response PDU. 


\section{Referências}

[An87] ANSI X3T5/87-305: Tutorial Material for Development of OSI Management Model Section V - Information Model, Novembro de 1987.

[Ad96] Advent Network Management, Inc.; Advent SNMP Java API; http://www.advent.com/index.html, 1996.

[Be90] Besaw, L. et al.,The Common Management Information Services and Protocols for Internet. (DDS Network Information Center, SRI International), Request for Comment 1095, 1990.

[Be95] Bem-Natan, R., A guide to Common Object Request Broker Architecture, McGraw Hill, 1995.

[Bo91] Booch, G.: Object Oriented Design with Applications. The Benjamin/Cummings Publishing Co., Inc., 1991.

[Bo95] Boisseau, B., Demange, M., Munier, J.M., An Introduction to ATM Technology. International Thompson Computer Press, 1995.

[Ca93] CASE, J. et. al. Manager-to-Manager Management Information Base. SNMP Research, Inc., Hughes LAN System, Dover Beach Consulting, Inc., Carnegie Mello University, Request for Comments 1451, 1993

[Ca93a] Case, J. et. al. Introduction to version 2 of the Internet-standard Network Management Framework. SNMP Research, Inc., Hughes LAN System, Dover Beach Consulting, Inc., Carnegie Mello University, Request for Comments 1441, 1993.

[Co94] Computer Systems Research Group: System Manager's Manual. Berkeley Software Distribuition, University of California at Berkeley. USENIX / O'Reilly \& Associates, Inc., 1994 
[Da87] Davin, J. et al., Simple Gateway Monitoring Protocol. (DDS Network Information Center, SRI International), Request for Comment 1028, 1987.

[Da93] Davin, J.; McCloghrie, K., Administrative Model for version 2 of the Simple Network Management Protocol (SNMPv2); (DDS Network Information Center, SRI International), Request for Comment 1445, 1993.

[Da93a] Davin, J.; McCloghrie, K.; Security Protocols for version 2 of the Simple Network Management Protocol (SNMPv2); (DDS Network Information Center, SRI International), Request for Comment 1446, 1993.

[Da97] Dave, R.; HyperText Markup Language v3.2; World Wide Web Consortium; http://www.w3.org/pub/WWW/TR/REC-html32.html, 1997.

[De96] Deri, L. Surfin; Network Resources across the Web, IBM Corp., http://misa.zurich.ibm.com/Webbin/ Ide/Surfin.ps.Z, 1996.

[Ea95] Earnshaw, R. A.; Vince, J. A.; Multimedia Systems \& Applications; Academic Press, 1995.

[Fi97] Fielding, R, et al; Hypertext Transfer Protocol (HTTP/1.1), (DDS Network Information Center, SRI International), Request for Comment 2068, 1997.

[Fr96] Freir, A., O.; Karltan, P.; Kacher, P. C.; The SSL Protocol version 3.0; Transport Layer Security Working Group, (DDS Network Information Center, SRI International) ftp://ds.internic.net/internet-drafts/draft-ietf-tls-ssl-version3-00.txt, 1996.

[Go96] Gosling, J.; Joy, B.; Steele, G.; The Java Language Specification; AddisonWesley, 1996.

[Is89] ISO 7498-4 - Information Processing Systems - Open System Interconnection - Basic Reference Model - Part 4: Management Framework, Geneva, 1989. 
[Is90] ISO 9595 - Information Processing Systems - Open System Interconnection Common Management Information Service Definition, Geneva, 1990;

[Is92] ISO 10040 - Information Processing Systems - Open System Interconnection - System Management Overview, Geneva, 1992;

[Is92a] ISO DP 9596/1,2 - Information Processing Systems - Open System Interconnection - Management Information Protocol Specification, 1992;

[Is93] ISO DIS 10165 - Information Processing Systems - Open System Interconnection - Structure of Management Information - Part 1: Management Information Model, Geneva, 1993.

[Jo88] Joseph, C.A; Muralidhar, K. H.: Network Management: A Manager's Perspective. ENTERPRISE Conf. Proc., pg 5-163-5-174, Baltimore, MD, junho de 1988.

[Jo90] Joseph, C.A; Muralidhar, K. H.: Integrated Network Management in na Enterprise Enviroment. IEEE Network, julho de 1990.

[K188] Klerer, S. M.: The OSI Management Architecture: An Overview. IEEE Network, Vol. 2, No. 2, pg. 20-29, Março de 1988.

[Ku92] Kurose, J.: Open Issues and Challenges in Providing Quality of Service Guarantees in High-Speed Networks. 3rd International Workshop on Network and Operating System Support for Digital Audio and Video, San Diego, 1992.

[Li95] Lieira, J., Utilização de Vídeo e Áudio em Sistemas de Gerenciamento de Redes de Computadores. Dissertação de Mestrado, ICMSC-USP, 1995.

[Li97] Lindholm, T., et al., The Java Virtual Machine Specification, AddisonWesley, 1997. 
[Mc91] McCloghrie, K.; ROSE, M. T., Management Information Base for Network Management of TCP/IP-based internets: MIB-II. (DDS Network Information Center, SRI International), Request for Comment 1213, 1991.

[Mo94] Moreira, E. S.; Oda, C. S.; Cicilini, R., Desenvolvimento de um Gerenciador de Redes de Computadores Com Recursos de multimídia. SEMINFO, Salvador, Bahia, 1994.

[Mu89] Müllender, S. J.: Distributed Systems. ACM Press, 1989.

[Od94] Oda, C. S., Desenvolvimento de um Sistema Monitor Gráfico Baseado em Protocolo de Gerenciamento SNMP. Disertação para obtenção do título de Mestre na área de Ciências de Computação e Matemática Aplicada - USP/ICMSC - São Carlos, 1994.

[Od95] Oda, C. S.; Moreira, E. S., Representação Dinâmica de Objetos em Ambientes de Gerenciamento de Redes de Computadores. Proceedings 130. Simpósio Brasileiro de Redes de Computadores, Belo Horizonte, maio/1995.

[Od95a] Oda, C. S.; Moreira, E. S., An X-Window based Monitor with Intelligent Topology Discovery. Proceedings ISSM International Conference on Intelligent Management, Washington DC, USA, junho/1995.

[Po80] Postel, J., User Datagram Protocol (UDP). (DDS Network Information Center, SRI International), Request for Comment 768, 1980.

[Pr95] Pras, Aiko - Network Management Architectures. CTIT Ph.D-thesis University of Twente, Enschede, 1995.

[Ro90] Rose, M. T., Structure and Identification of Management Information for TCP/IP-based internets. (DDS Network Information Center, SRI International), Request for Comment 1155, 1990 
[Ro91] Rose, M. T., The Simple Book - An Introduction to Management of TCP/IP-based Internets. Englewood Cliffs, Prentice-Hall, 1991.

[Ro91a] Rose, M. T.; McCloghrie, K., Concise MIB Definition. (DDS Network Information Center, SRI International), Request for Comment 1212, 1991.

[Sc90] Schoffstall, M. et. al., A Simple Network Management Protocol (SNMP). (DDS Network Information Center, SRI International), Request for Comment 1157, 1991.

[St93] Stallings, W., SNMP, SNMPv2, and CMIP - The Pratical Guide to NetworkManagement Statandards. Addison-Welley, 1993.

[Su88] Sun Documentation, SunOS Reference Manual. Sun Microsystems, 1988.

[Sw96] Swerdlow, J. F., A Revolução da Informática: Da Auto-Estrada de Dados Eletrônicos ao Mundo da Comunicação Virtual. Deutschland, Frankfurter Societäts-Druckerei GmbH, D20012F, No. 1, Fevereiro de 1996.

[Tr87] Trewitt, G.; Partridge, C., High-Level Entity Management System (HEMS). (DDS Network Information Center, SRI International), Request for Comment 1021, 1987.

[Tr87a] Trewitt, G.; Partridge, C., High-Level Entity Management Protocol (HEMP). (DDS Network Information Center, SRI International), Request for Comment 1022, 1987.

[Wa95] Waldbusser, S., Remote Network Monitoring Management Information Base (RMON). (DDS Network Information Center, SRI International), Request for Comment 1757, 1995. 